\title{
James Webb Space Telescope (JWST) optical telescope element and integrated science instrument module (OTIS) cryogenic test program and results
}

Randy A. Kimble, Lee D. Feinberg, Mark F. Voyton, Juli A. Lander, J. Scott Knight, et al.

Randy A. Kimble, Lee D. Feinberg, Mark F. Voyton, Juli A. Lander, J. Scott Knight, Mark Waldman, Tony Whitman, M. Begoña Vila Costas, Carl A. Reis, Kan Yang, "James Webb Space Telescope (JWST) optical telescope element and integrated science instrument module (OTIS) cryogenic test program and results," Proc. SPIE 10698, Space Telescopes and Instrumentation 2018: Optical, Infrared, and Millimeter Wave, 1069805 (24 July 2018); doi: 10.1117/12.2309664

Event: SPIE Astronomical Telescopes + Instrumentation, 2018, Austin, Texas, United States 


\title{
James Webb Space Telescope (JWST) optical telescope element and integrated science instrument module (OTIS) cryogenic test program and results
}

\author{
Randy A. Kimble*a, Lee D. Feinberg ${ }^{\mathrm{a}}$, Mark F. Voyton ${ }^{\mathrm{a}}$, Juli A. Lander ${ }^{\mathrm{a}}$, J. Scott Knight ${ }^{\mathrm{b}}$, Mark \\ Waldman $^{\mathrm{c}}$, Tony Whitman ${ }^{\mathrm{d}}$, M. Begoña Vila Costas ${ }^{\mathrm{e}, \mathrm{a}}$, Carl A. Reis ${ }^{\mathrm{a}}$, and Kan Yang ${ }^{\mathrm{a}}$ \\ aNASA's Goddard Space Flight Center, 8800 Greenbelt Rd, Greenbelt, MD 20771 \\ bBall Aerospace \& Technologies Corporation, 1600 Commerce St, Boulder, CO 80301 \\ ${ }^{\mathrm{c}}$ Sigma Space Corporation, 4600 Forbes Blvd., Lanham, MD 20706 \\ ${ }^{\mathrm{d}}$ Harris Corporation, 400 Initiative Drive, Rochester, NY 14624 \\ eStinger Ghaffarian Technologies, 7701 Greenbelt Rd \#400, Greenbelt, MD 20770
}

\begin{abstract}
In 2017, the James Webb Space Telescope Optical Telescope Element and Integrated Science Instrument Module (OTIS) underwent cryogenic optical testing at the Johnson Space Center. In this paper, we summarize the successful execution and results of this 100-day test, which was a major program milestone. We summarize the as-run test configuration and provide a top-level as-run timeline. We also provide the top-level functional, optical, thermal, and operational results from the test. We summarize the key technical issues encountered and the resolution of those issues. The results of the OTIS test indicate that the payload should be fully capable of delivering on JWST's exciting scientific potential.
\end{abstract}

Keywords: telescope, infrared, cryogenic, testing, JWST, ISIM, OTE, OTIS

\section{INTRODUCTION}

\subsection{Introduction to the OTIS}

The James Webb Space Telescope (JWST) is a cryogenic, large-aperture (6.5m diameter), segmented-telescope observatory, in development to carry out forefront astronomical observations over a range of near- and mid-infrared wavelengths from 0.6 to $28 \mu \mathrm{m}$. See McElwain et al. $(2018)^{1}$ in this volume for an overview of the project and its current status. At this time, the integration of the observatory has funneled down to two principal sub-elements: the integrated sunshield and spacecraft, known as the Spacecraft Element (SCE), currently in integration and test at Northrop Grumman Aerospace Systems, and the combination of the Optical Telescope Element (OTE) and the Integrated Science Instrument Suite (ISIM), which, as an assembly, is known as the OTIS (= OTE + ISIM). OTIS testing is the subject of this paper.

The Optical Telescope Element is comprised of the three-mirror anistigmat telescope (with its 18-segment Primary Mirror) and Fine Steering Mirror, along with the composite structures to support those optics and the mechanical and electrical systems required to deploy them (including the Secondary Mirror Support System, two 3-segment deployable wings for the Primary, and the actuators for deploying [from their launch restraints] and aligning the Primary Mirror segments and Secondary). Integration of the OTE was completed in 2016, as described by Atkinson et al. (2016) ${ }^{2}$.

The Integrated Science Instrument Module (ISIM) for the James Webb Space Telescope (JWST) is comprised of four scientific instruments (SIs) and various structural, thermal, electronic, and operational systems that support them. The SIs are: the Near-Infrared Camera (NIRCam) ${ }^{3,4}$, the Near-Infrared Spectrograph (NIRSpec) ${ }^{5,6}$, the Mid-Infrared Instrument (MIRI) ${ }^{7.8}$, and the Fine Guidance Sensor/Near-Infrared Imager and Slitless Spectrograph (FGS/NIRISS) ${ }^{9,10}$. Within the latter module are both a science channel (NIRISS) and a pair of Fine Guidance Sensor channels which, as the name implies, provide pointing error signals on selected guide stars to the attitude control system for the observatory. The flight OTE and ISIM hardware are shown in Figure 2.

*randy.a.kimble@nasa.gov; phone 1301 286-5783

Space Telescopes and Instrumentation 2018: Optical, Infrared, and Millimeter Wave, edited by Makenzie Lystrup,

Howard A. MacEwen, Giovanni G. Fazio, Proc. of SPIE Vol. 10698, 1069805

(C) 2018 SPIE · CCC code: 0277-786X/18/\$18 · doi: 10.1117/12.2309664 


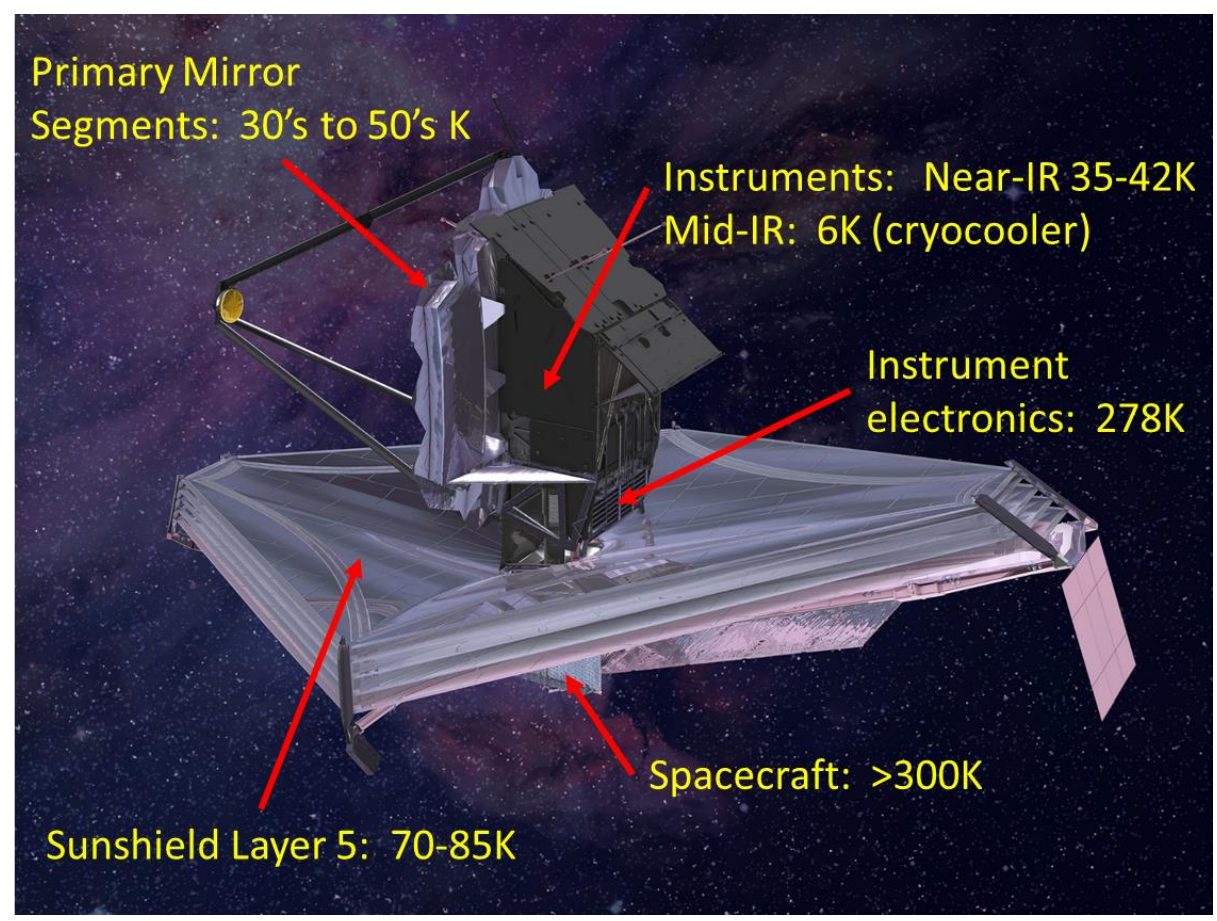

Figure 1. The JWST architecture, in which the cryogenic temperatures required for sensitive infrared observations are achieved by hiding the science payload in the shadow of a five-layer sunshield, in a halo orbit around the Sun-Earth L2 point, 1.5 million km from Earth. The OTIS (whose test program is the subject of this paper) is essentially everything above the plane of the upper sunshield layer in this diagram. The Spacecraft Element is everything from the sunshield down.
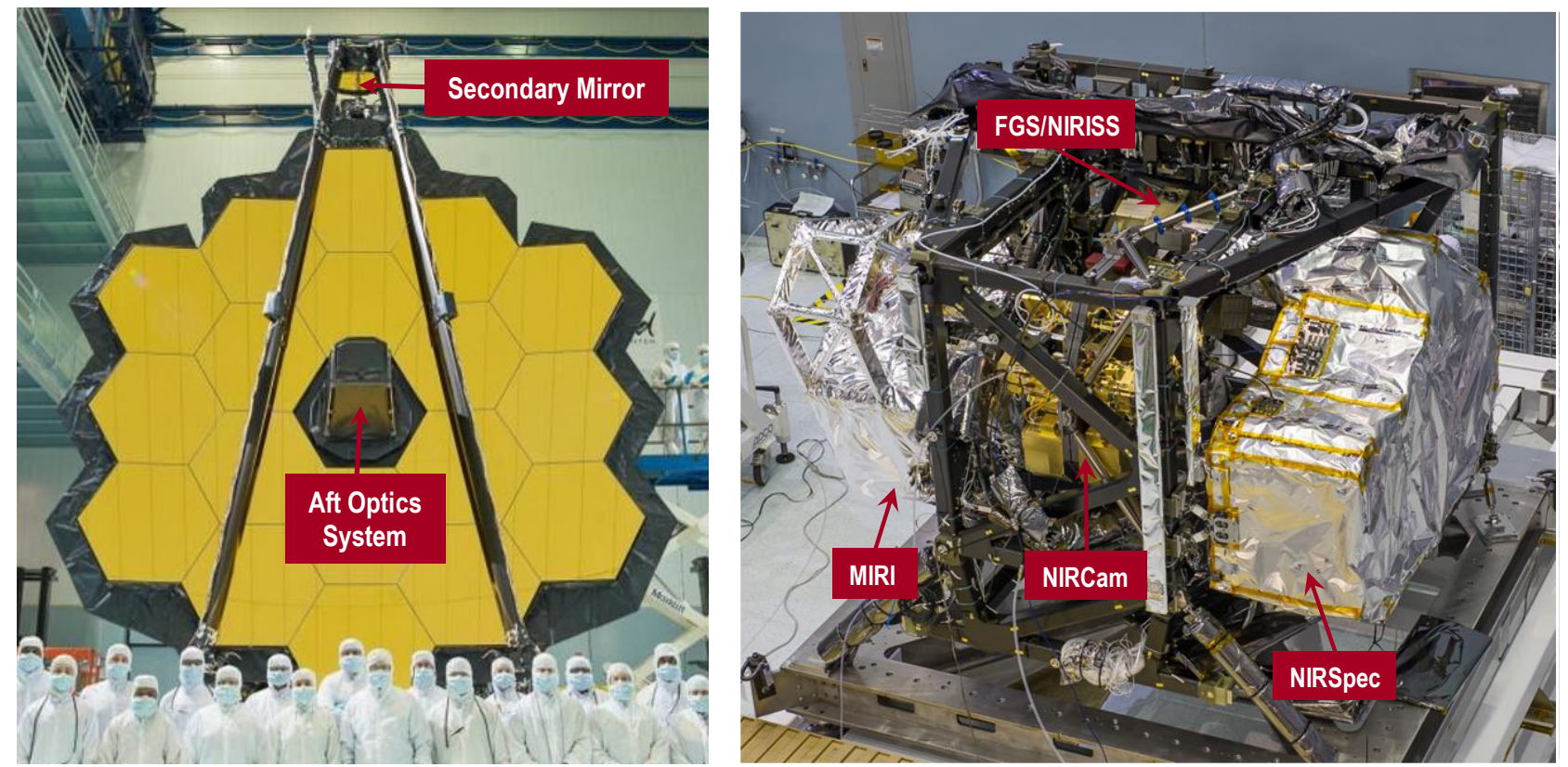

Figure 2. The hardware elements making up the OTIS. Left) The Optical Telescope Element with its 18-segment Primary Mirror and the Aft Optics System (which holds the Tertiary Mirror and Fine Steering Mirror) in the center. The telescope wings (in their deployed state) are the three-segment vertical sections at the left and right. The Secondary Mirror Support System is shown in its stowed configuration. Right) The four ISIM science instruments, mounted to a highly stable composite metering structure. The ISIM also includes heat straps and a large near-room-temperature electronics assembly (the ISIM Electronics Compartment, or IEC), whose location is shown schematically in Figure 1. 


\subsection{Context and objectives for the OTIS cryo-vacuum test}

Integration of the Optical Telescope Element was completed at Goddard Space Flight Center (GSFC) in early 2016, essentially simultaneously with the completion and delivery of the Integrated Science Instrument Module (after its final element-level cryo-vacuum test program ${ }^{11}$ ). These two elements were then integrated in the GSFC cleanroom to form the OTIS. After the execution of a Center of Curvature optical test of the Primary Mirror ${ }^{12}$, as well as various functional and deployment tests (e.g. of the telescope wings, Deployable Tower Assembly, and the Aft Deployable ISIM Radiator) the OTIS payload then entered its ambient environmental test program. These included sine-vibration and acoustic testing at proto-flight levels, which were then followed by repeats of the optical, functional, and deployment checks.

The ambient-tested OTIS next was delivered to the Johnson Space Center (JSC) in early 2017 and prepared for cryovacuum testing in that facility's historic Chamber A. See Section 2 (below) for a description of the test architecture and the sophisticated hardware that was developed to support it.

The OTIS cryo-vacuum (CV) test represented a huge milestone in the JWST program. OTIS is the highest level of assembly at which the telescope and instruments are cryo-tested - when the OTIS is mated to the Spacecraft Element to form the full-up JWST Observatory, that assembly will be vibration and acoustic tested (at acceptance, not proto-flight, levels), but not cryo tested. Hence, the OTIS CV was an exceptionally significant event, and as the last stage of OTIS environmental testing, the OTIS CV test was designed to accomplish a wide variety of important objectives:

a) Functional tests/health checks. As noted, the OTIS CV campaign took place after the vibration and acoustic testing of the OTIS hardware. It thus represented the opportunity to confirm that the hardware came through that exposure in good health and with proper functionality in flight-like cryo operations. The ISIM element had been through several test campaigns previously, but OTIS CV was the only cryo-test of the integrated OTE. Comprehensive functional tests both at ambient vacuum and at operational cryo-temperatures were thus key parts of the OTIS CV program.

b) Optical verifications and cross-checks. The largest portion of the cryo-stable phase of the campaign was devoted to optical testing. Formal requirement verifications included: critical alignments (PM to AOS; SM to AOS; ISIM to AOS - where PM = Primary Mirror; SM = Secondary Mirror; AOS = Aft Optics System [Tertiary Mirror + Fine Steering Mirror]); pupil alignments; PM radius of curvature; verification of Wavefront Sensing and Control operations.

A wide variety of additional optical measurements provided cross-checks of important requirements. In these cases, the formal verification path for a given requirement may utilize measurements from a lower level of integration, where more precise measurements were feasible than in the full-up OTIS test (e.g. figure/Wavefront Error (WFE) measurements for individual optics). However, the full-up measurements provided valuable confirmation, at a meaningful precision, that nothing had gone badly awry in the build up to the final assembly. These cross-checks included: multi-field assessments of PM and SM alignment to AOS; PM and AOS Wavefront Error cross-checks, over a range of spatial scales; PM conic; thermal distortion checks of PM WFE and radius of curvature changes or overall alignment shifts with changing payload temperature; PM collection area; vignetting; mirror actuator repeatability and mirror envelope control.

c) Thermal objectives. The most fundamental thermal objective was to exercise the payload through the challenging cooldown to its stable, flight-like cryogenic operating temperatures (where functional, optical, thermal, and operational tests would take place) and then back up to room temperature, while keeping the hardware safe from structural and contamination standpoints. At the cryo-stable operating conditions, the highest priority thermal goal was then to establish a stable thermal balance at flight-like temperatures for model validation. Additional objectives included confirming the health and workmanship of thermal hardware (sensors, heaters, heat straps) and making heat load measurements and leak checks relevant to the mechanical cryo-cooler for the Mid-Infrared Instrument (MIRI).

d) Mechanical objectives. Some major deployable elements of the OTE (the Primary Mirror wings, the Secondary Mirror Support System) were already deployed before the test, as they require complex offloading for 1-g operation. However, mechanical tests included drive motor current trending and flinch tests for deployment systems, pre-load measurements using strain sensors, as well as deployment motor heater tests relevant to flight contingency scenarios.

e) Operational objectives. Operational objectives included closed-loop testing of the interaction between the Fine Guidance Sensors and the Fine Steering Mirror (functional, parity, and timing checks; the only pre-flight opportunity, hence absolutely critical), and extensive exercising of the Onboard Script System (OSS), including checkout of new or revised instrument scripts, OTE script operations (mirror control software, Wavefront Sensing \& Control script demonstrations); Day-in-the-Life testing with flight-like operational scenarios. Calibration reference data (e.g. detector dark ramps) of future use for flight operations were also acquired as opportunities permitted. 


\section{OTIS CRYO-VACUUM TEST CONFIGURATION}

The venue for accomplishing these varied test objectives was the historic Chamber A at Johnson Space Center in Houston, Texas. Chamber A is a U.S. National Historic Landmark, stemming from its use during the Apollo program. For JWST cryo-testing, the chamber was cleaned and substantially refurbished - and outfitted with a high-performance helium cooling shroud for achieving JWST-required temperatures, with an adjoining cleanroom for pre- and post-test operations with the contamination-sensitive payload.

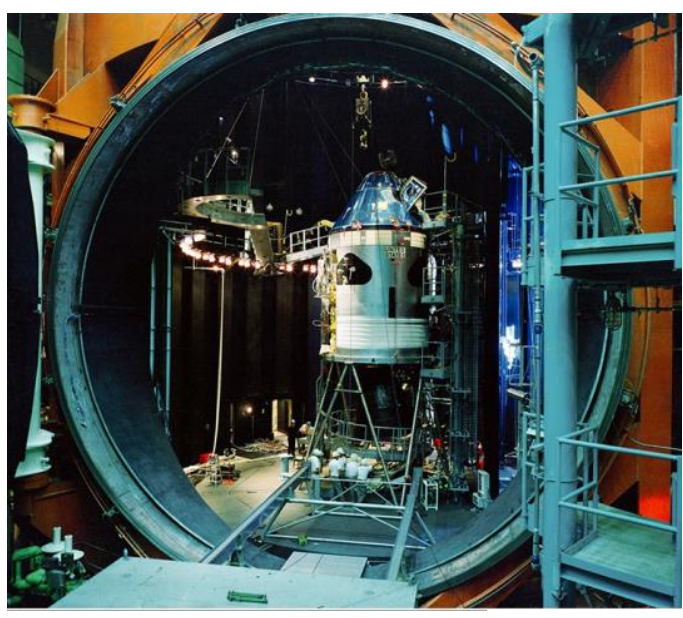

Figure 3. Chamber A at JSC.

Left) Setting up to test a Command Module in the Apollo era

Right) About to receive the JWST OTIS hardware for its cryo-vacuum test campaign.

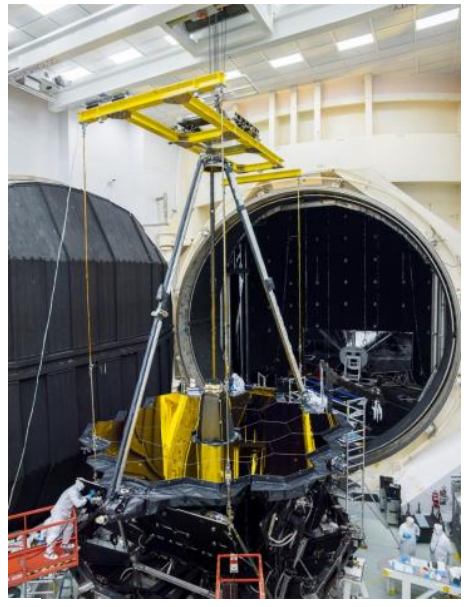

Vibration Isolators (6)

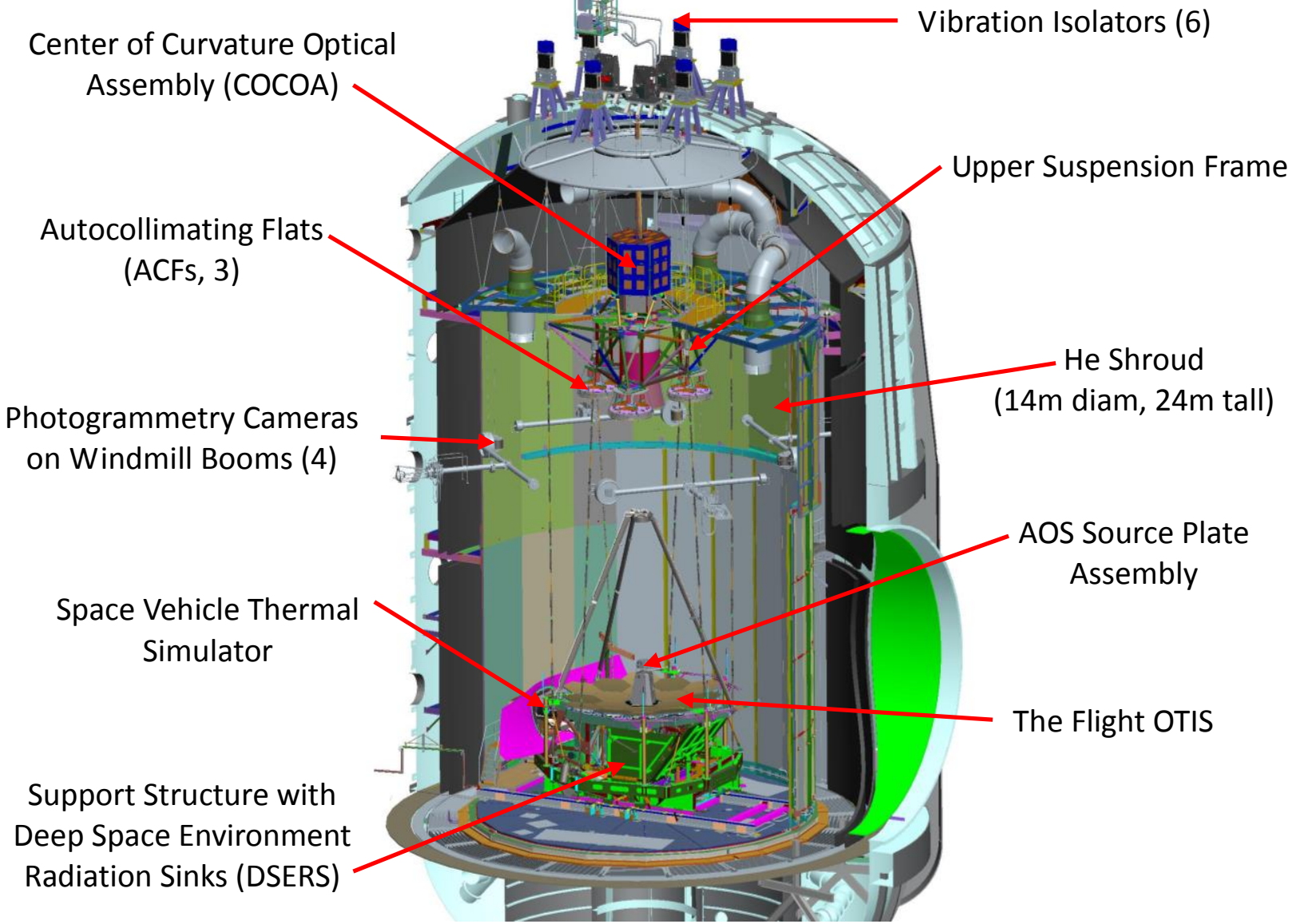

Figure 4. The hanging test configuration for the OTIS cryo-vacuum test at Johnson Space Center. See the text below for more details of the test hardware highlighted in the Figure. Most of the GSE pictured here was developed by Harris, Inc. 


\subsection{Overview of the ground support equipment (GSE)}

As shown schematically in Figure 4, the OTIS payload was mounted on a support structure that was suspended from the top of the chamber by a set of long rods that coupled ultimately to a suite of six vibration isolators mounted on top of the chamber. These isolators damped the vibration of the suspended assembly and could be adjusted from outside the chamber. Also part of the hanging assembly was an Upper Suspension Frame, which supported two critical pieces of optical GSE: the Center of Curvature Optical Assembly (COCOA) and a set of three 1.5m-diameter Autocollimating Flat mirrors (ACFs).

The COCOA, illustrated in Figure 5, consisted of a Multiple-Wavelength Interferometer (MWIF), a reflective null, a MWIF null calibration system, coarse and fine alignment systems, and Displacement Measuring Interferometers (DMIs). The MWIF and DMIs were mounted in a pressure-tight enclosure. A hexapod support system permitted positioning of the COCOA in six degrees-of-freedom. The COCOA resided above the He cooling shroud of the chamber in an LN2 environment, but was maintained at room temperature; a cold shutter isolated the COCOA from the OTIS and test hardware in the He shroud when not in use.

With the shutter open, the COCOA had an unobstructed view of the entire Primary Mirror and was used to align and phase the $18 \mathrm{PM}$ segments. An Absolute Distance Meter mounted below the PM used targets mounted to the PM and to the COCOA to set the COCOA's axial distance, enabling measurements of the PM radius of curvature. See Wells et al. (2010) ${ }^{13}$ and Hadaway et al. (2016) ${ }^{14}$ for further details of the

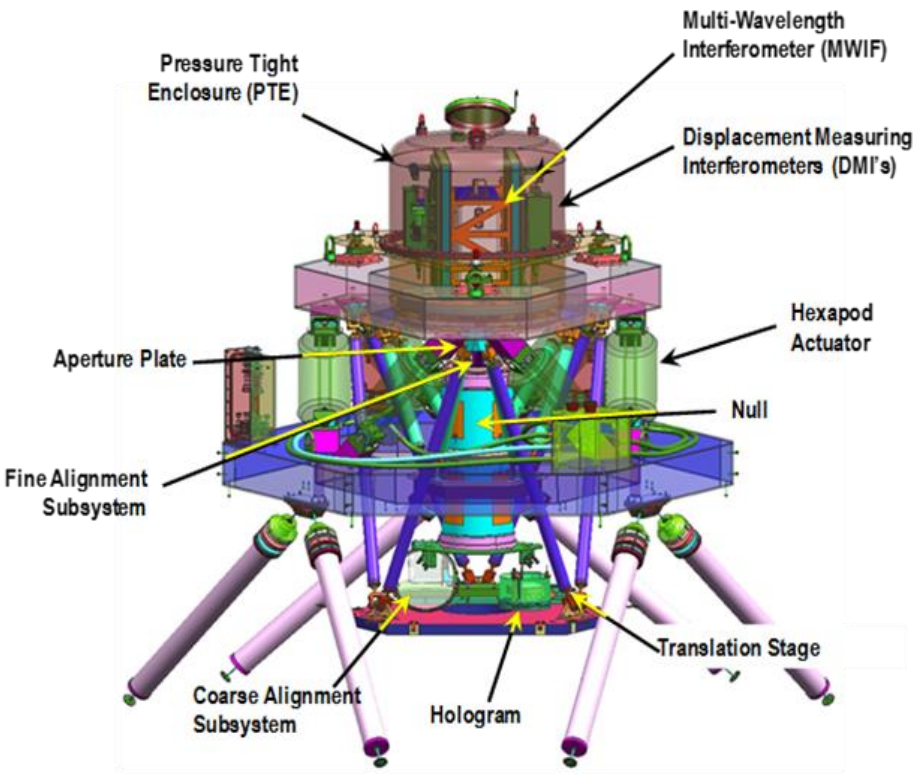

Figure 5. The Center of Curvature Optical Assembly. See text at left for details. design and use of the COCOA.

The schematic in Figure 4 shows, farther down in the chamber, the location of four windmill booms to which four photogrammetry (PG) cameras were mounted. As the booms were rotated during a PG "run", the cameras took images that captured the positions of targets mounted to the OTIS flight hardware and GSE at strategic locations, including coded targets and reference scale bars (whose dimensions had previously been precisely calibrated at the relevant temperatures). The suite of images from a given run was analyzed with a commercial software package to yield, through triangulation, the 3-D positions of all the targets within the test volume, to remarkable accuracy. This capability was used to perform rough alignment of the adjustable PM and SM mirrors, guiding the adjustment of the mirror actuators.

The critical pieces of optical GSE described above were developed by Harris, Inc. Another vital piece of optical GSE, the AOS Source Plate Assembly (ASPA), was developed by Ball Aerospace (see Knight et al. [2014] ${ }^{15}$ ). This hardware, shown in Figure 6, was mounted, as the name implies, to the top of the Aft Optics System, at the Cassegrain pseudofocus of the three-mirror anistigmat JWST optical system. Numerous small light sources were fiber-fed to or located at that image plane. Some were aimed inward toward the Tertiary Mirror and Fine Steering Mirror (FSM) and ultimately the science instruments; this was the so-called Half-Pass (HP) light path. Other sources were aimed outward toward the Secondary Mirror. From there, the light reflected back to the Primary and up to the three sub-aperture ACFs mounted high in the chamber. The return from those ACFs illuminated 9 of the 18 PM segments and propagated through the rest of the optical system to the instrument focal planes, in what was termed the Pass-and-a-Half (PAAH) light path.

As the Cassegrain focus is not a perfect image plane, the resulting images from both of these paths are highly aberrated. Nonetheless, as those aberrations are perfectly modelable, the images obtained can be compared to predictions to support a wide variety of optical testing goals, both critical alignment verifications and a number of cross-checks and demonstrations. 
a)

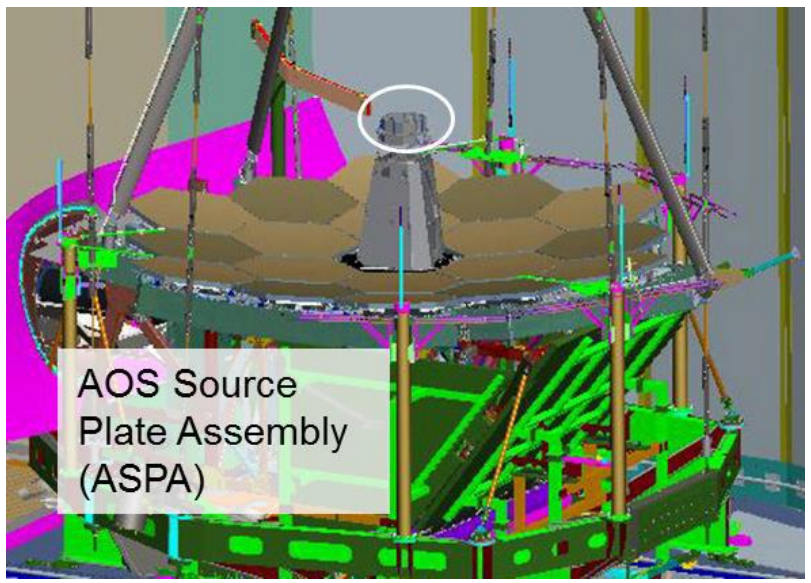

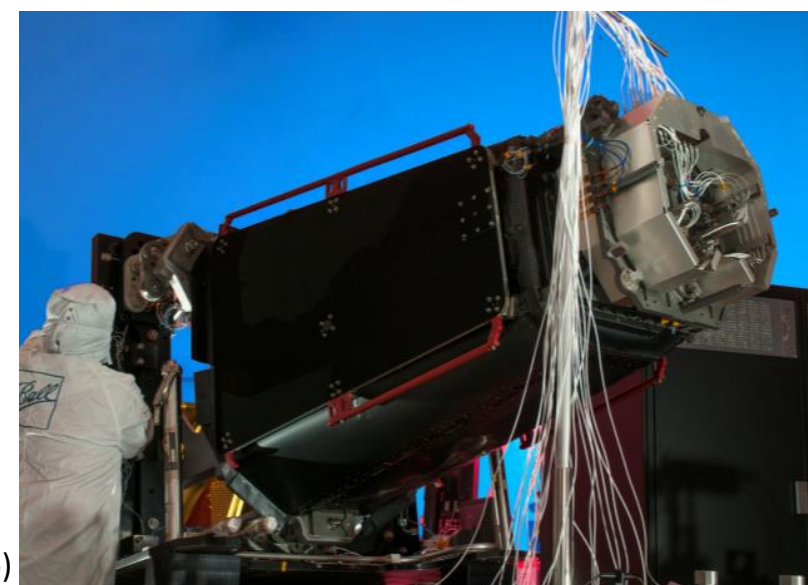

Figure 6.

a) Schematic location of the AOS Source Plate Assembly

b) Photo of the flight AOS and the ASPA, showing the fiber-bundle that feeds in light from sources outside the chamber

c) Schematic of the fiber inputs to the various positions in the field of view

d) Photo of the as-built hardware.

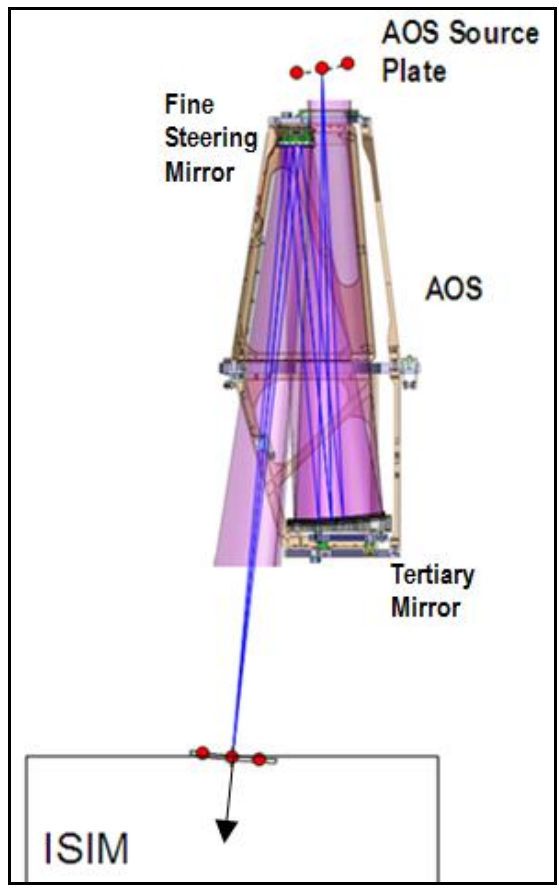

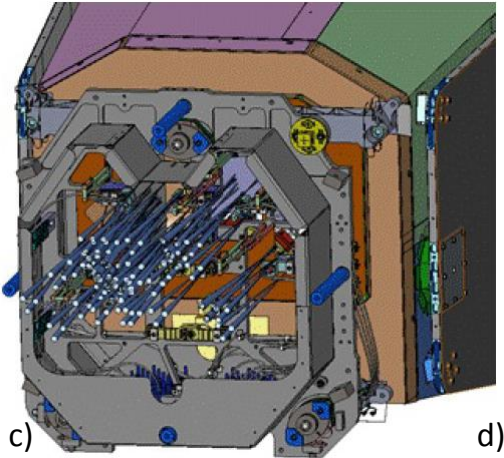

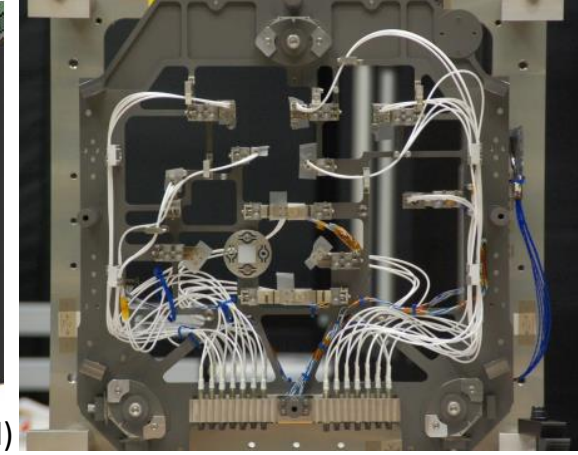

Figure 7

Left) The Half-Pass (HP) light path, in which downward facing sources propagate via the Tertiary and Fine Steering Mirror to the science instrument focal planes.

Right) The Pass-and-a-Half (PAAH) light path, in which upward facing sources propagate via the SM and PM to the ACFs and then back through the entire optical system to the instrument focal planes.

As the diagram indicates, the PAAH configuration provides sub-aperture illumination, covering portions of 9 of the 18 PM segments.

Note also that the ACFs had fine tip/tilt actuation, permitting their returns to be coaligned or not, as test goals required.

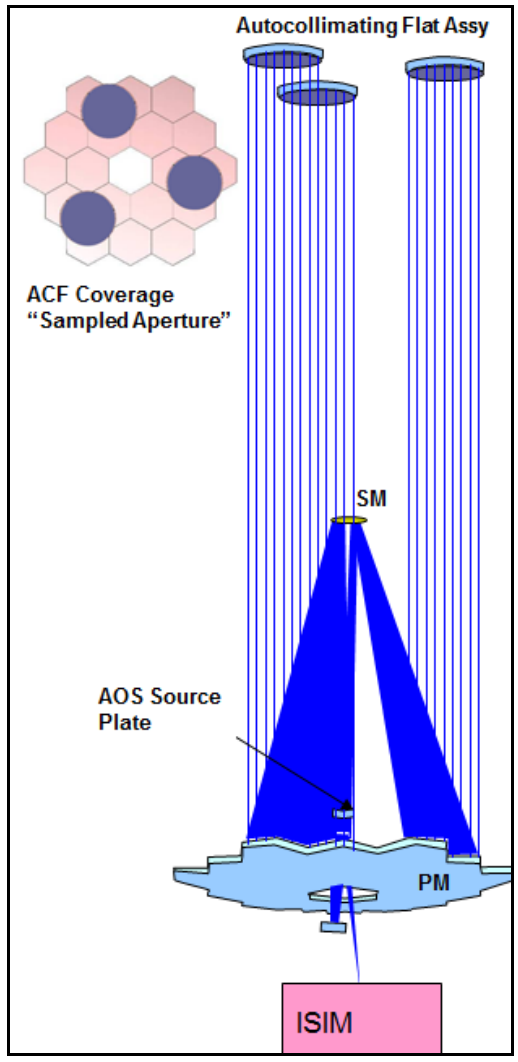


The thermal GSE was absolutely crucial for achieving the test objectives for this cryogenic payload, of course. Details of the thermal GSE and its operation can be found in Yang et al. (2018) ${ }^{16}$. First and foremost are the chamber's LN2 and He shrouds; the latter was ultimately cooled to $20 \mathrm{~K}$ to serve as the cold sink for the flight and test hardware. Other key thermal GSE (developed principally by Harris, Inc.) included:

- The Space Vehicle Thermal Simulator (SVTS). This assembly (see the schematic in Figure 4) emulated the thermal properties and interfaces of the core region where the Deployable Tower Assembly and electrical harnesses of the OTIS interface through the sunshield to the warm spacecraft. For the sunshield itself, analysis determined that only the warmer, inner regions of the sunshield's telescope-facing layer needed to be emulated for the purposes of this test. It would have been extremely difficult (and not required by test goals) to actively heat a full-up sunshield to its flight temperature profile, and almost impossible to achieve the correct geometric shape in 1-g for flight-like reflections.

- The Deep Space Environment Radiation Sinks (DSERS). Thermal control of the payload in flight relies on efficient radiation of the power dissipated on the telescope side of the sunshield by the OTIS radiators, in particular the elegant directional radiator designed to remove the large amount of power from the warm ISIM Electronics Compartment (IEC). The DSERS provided controlled high-emissivity sinks for this power. A system of interlinked DSERS panels surrounded the ISIM enclosure and radiators to achieve the correct thermal environment for the science instruments.

- Pre-cool straps and zero-Q heaters. Pre-cool straps provided thermal connectivity to various subsystems to enable cooldown to proceed on a faster timescale. Once desired operating temperatures were achieved, zero-Q heaters were then adjusted so that no net heat flowed to or from the flight payload along these non-flight-like paths.

- A GSE cryo-cooler (from JPL) was configured outside the chamber to serve the functions of the spacecraft portion of the MIRI cooler; the portion of the cooler hardware mounted to the OTIS payload was in the flight configuration.

\subsection{Use of the test architecture for optical testing}

The test architecture described above was the result of years of careful deliberation by the JWST team. More-broadlycapable, but harder-to-implement architectures had been considered, but were rigorously scrubbed to achieve a buildable, if-still-complex configuration that sufficed to achieve the essential verification and cross-check objectives. The process of arriving at the test setup ultimately chosen has been presented by Atkinson et al. (2008) ${ }^{17}$ and Feinberg et al. (2011) ${ }^{18}$.

A sense of how the optical pieces of the test configuration (see the simplified schematic in Figure 8) worked together to achieve the optical test goals is exemplified by the following succinct summary of the key features of the optical test program:

- Primary Mirror segments were aligned via Photogrammetry and COCOA interferometry.

- Secondary Mirror was aligned via Photogrammetry and Pass-and-aHalf imaging to the NIRCam instrument.

- AOS to ISIM alignment was verified via Half-Pass imaging using inward facing sources and all science instruments.

- Fiducial lights above the Primary Mirror were used for verifying pupil alignment, using NIRCam's pupil imaging capability.

- End-to-end imaging was cross-checked using Pass-and-a-Half imaging using outward facing sources, the Autocollimating Flats, and all of the science instruments.

- Wavefront Sensing \& Control hardware checks and demonstrations were performed via Pass-and-a-Half testing and NIRCam.

As the test unfolded, all of these elements worked together beautifully to achieve the desired purposes.

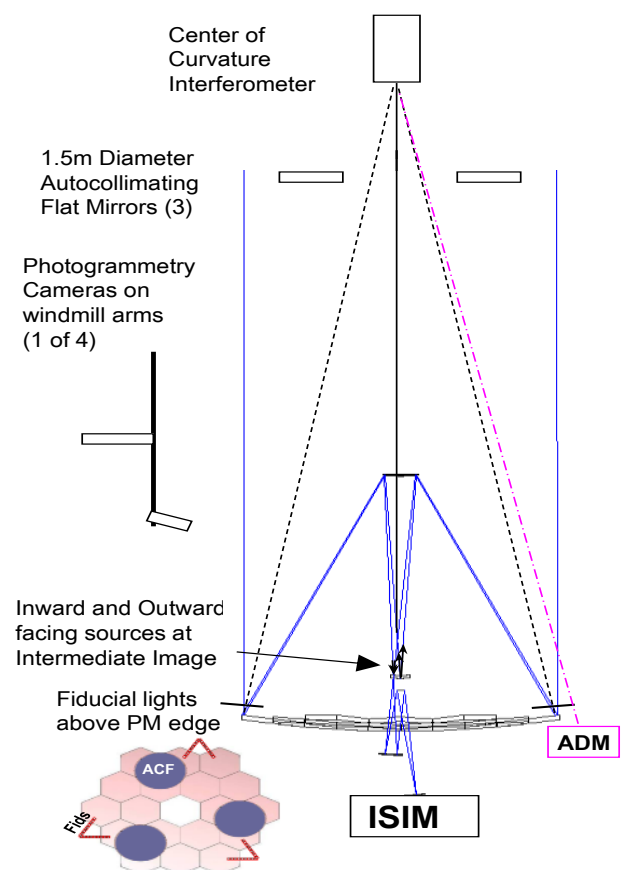

Figure 8. Simplified optical test schematic 


\subsection{The Pathfinder test program}

The elegant, but complex, test configuration and procedures described above could never have been implemented successfully in a single attempt, out of the box. It was recognized early on that it would be essential to check out all of this sophisticated GSE and dry-run procedures in a precursor test program, prior to installing the invaluable flight hardware into the setup. Accordingly, the Pathfinder test program was initiated to accomplish those goals. ${ }^{19,20}$

The key element of the Pathfinder program was a non-flight Primary Mirror backplane center section and Secondary Mirror Support System, provided for this purpose by Northrop Grumman. To this were mounted two spare Primary Mirror segments and the spare Secondary. As no spare Aft Optics System exists, one of the Pathfinder tests used the flight AOS itself to support and calibrate the ASPA assembly, in a carefully orchestrated integration and test flow that enabled the AOS to meet up with the rest of the flight OTE when the program scheduled required.

Ultimately, three Pathfinder cryo-vacuum tests were executed in Chamber A. Two focused on checkout of hardware and procedures with the optical GSE (and hence were designated OGSE1 and OGSE2); the third - Thermal Pathfinder checked out the thermal GSE and executed a representative cooldown profile with a higher-fidelity thermal emulation of the PM center section. Over the course of the Pathfinder program, hardware and software issues were corrected, procedures were dry-run, and invaluable experience was gained by the test team. Results of these efforts have been published regarding the performance of the Photogrammetry system ${ }^{21}$, the use of the $\operatorname{COCOA}^{22,23}$, chamber dynamics ${ }^{24}$, and the metrology of the ASPA sources ${ }^{25}$ (which was an essential precursor to their use in OTIS CV testing).

OGSE1: 2015, 32 days

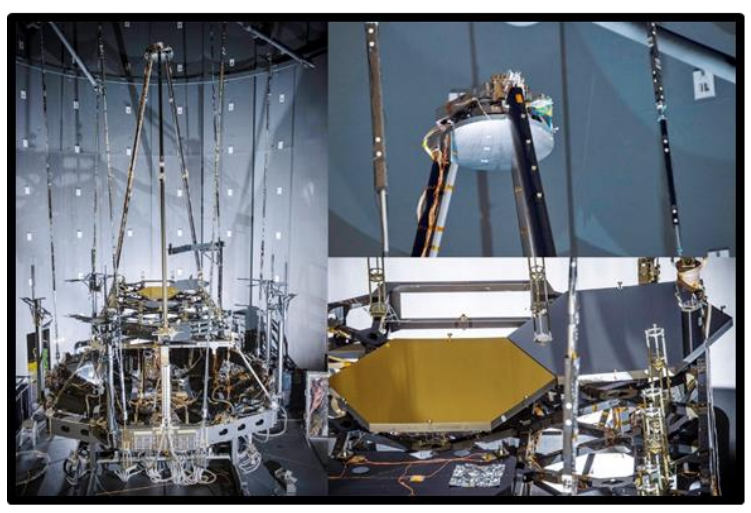

\section{OGSE2: 2015, 36 days}

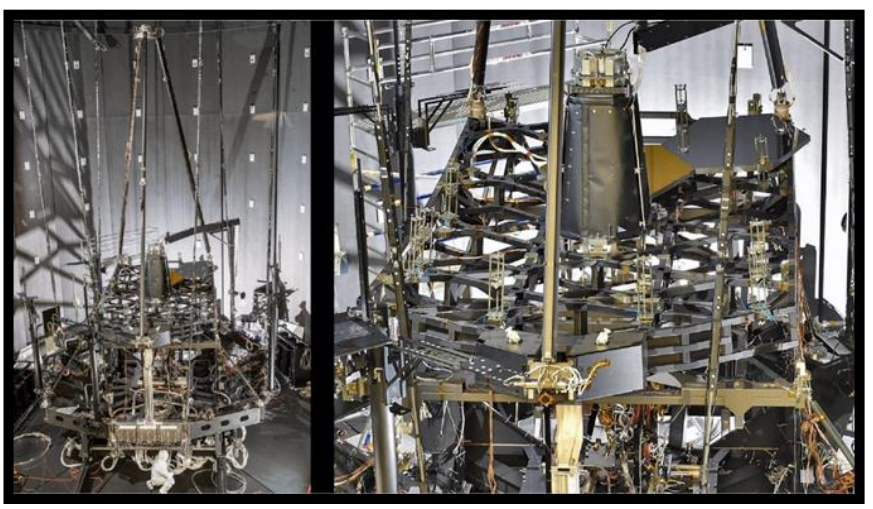

\section{Thermal Pathfinder: 2016, 54 days}

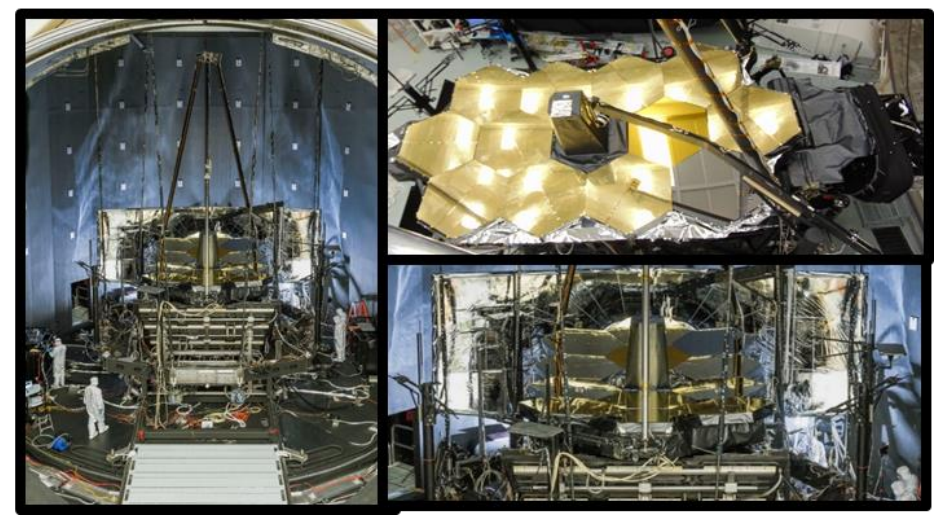

Figure 9. Pictorial summary of the Pathfinder test program, which provided invaluable experience with the complex optical and thermal GSE required to execute the OTIS cryo-vacuum test. The OGSE1 and OGSE2 focused on the use of the optical GSE with the two spare PM segments and SM, as well as the flight AOS (in OGSE2) for testing and calibration with the AOS Source Plate Assembly (ASPA). The Thermal Pathfinder test included thermal mockups of the rest of the 12 center-section PM segments and focused on checkout of the thermal GSE and procedures, though optical testing was carried out as well. 


\section{OTIS CRYO-VACUUM TEST EXECUTION}

\subsection{Schematic of the OTIS CV test timeline}

Meticulous preparations over a period of several months were required to ready the payload and GSE for vacuum operations. An important aspect of configuring for the test was the careful control of parasitic heat loads and in-band stray light entering the He shroud. Harnesses were heat-sunk outside the shroud. Warm sources were carefully enshrouded and light-leak paths minimized. After completion of these thermal and stray light preparations, the suspending of the payload, and electrical checks, the OTIS CV test began with chamber pumpdown on the $13^{\text {th }}$ of July, 2017. Round-the-clock test activities then proceeded for 100 days, until the backfill of the chamber on the $21^{\text {st }}$ of October. A summary timeline outlining the principal phases of the test activities is presented in Figure 10.

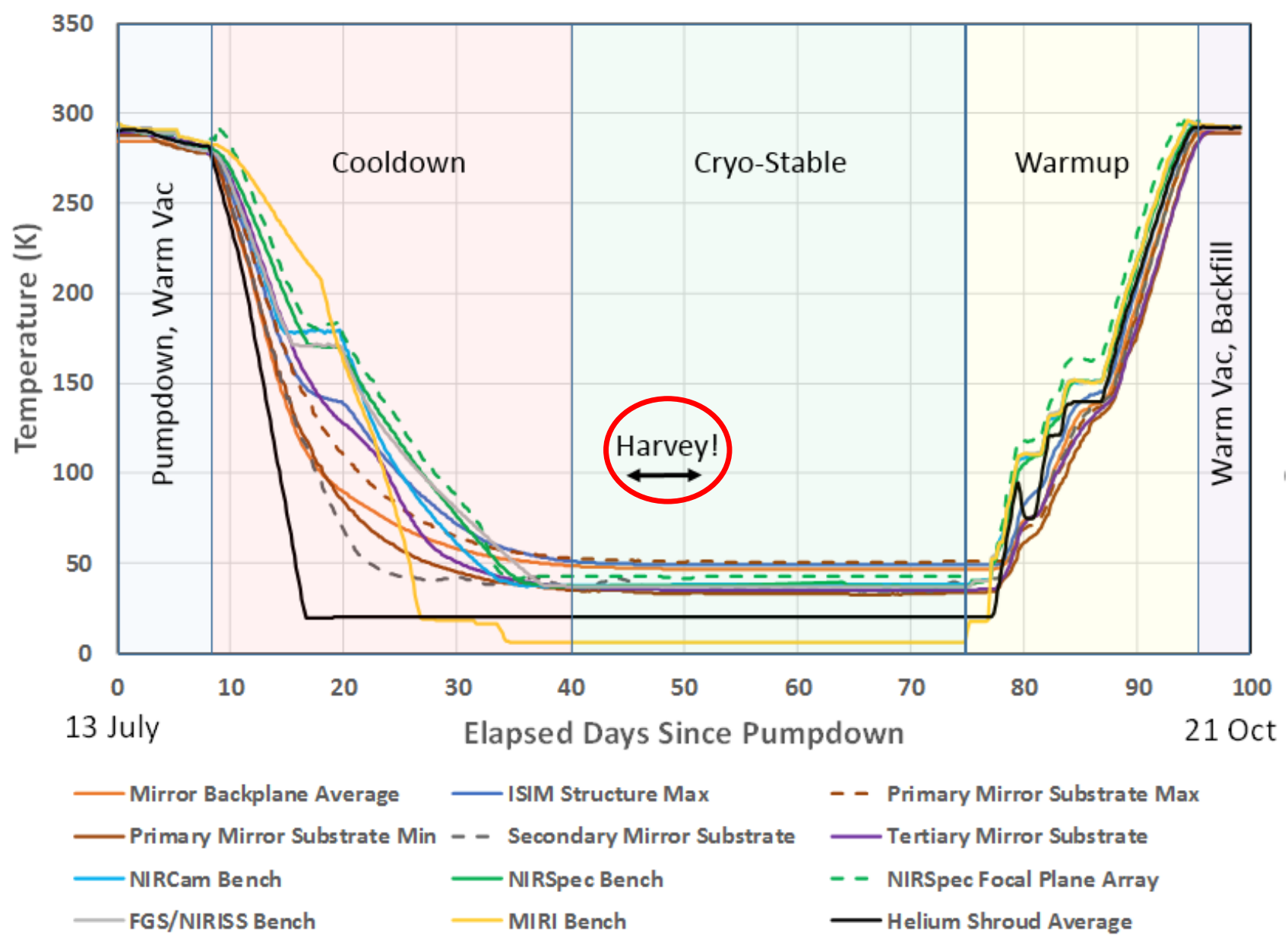

Figure 10. Summary of the as-run OTIS CV timeline, showing the temperature of key OTIS systems and the chamber He shroud, over the 100-day duration of the test. The principal phases of the test are highlighted - note the weeks required to cool down and warm up the payload safely - along with the time period of Hurricane Harvey's dramatic impacts on the JSC and Houston area.

\subsection{Chronological overview of the OTIS CV campaign}

In the sub-sections below, we highlight the principal test activities accomplished in the various phases of the OTIS CV campaign. The key results of those activities are then summarized in the next section. 


\subsubsection{Pumpdown and ambient temperature vacuum operations (days 0-8)}

Initial activities focused on vacuum operations: roughing the chamber and transitioning to hi-vacuum cryopumps. Leakchecking of the huge facility was carried out, and rate-of-rise measurements (pressure readings vs. time with the vacuum pumps isolated from the chamber) ultimately confirmed that the chamber had achieved the lowest leak rate of any JWST test, including the chamber commissioning runs and the Pathfinder program. When appropriate vacuum conditions were achieved, warm functional checkouts of both the OTE and the ISIM (as well as the optical and thermal GSE) were carried out to ensure that all electrical connections were good and that the payload was in good health in preparation for cooldown. Payload outgassing measurements were made during the warm vacuum period and met requirements.

Initial photogrammetry (PG) runs were made to establish initial positions of the flight hardware and GSE. In parallel with the execution of the ISIM warm functional, the 12 PM segments of the outer ring were deployed from their launch restraints. Nominal deployments are $12.5 \mathrm{~mm}$ for each of the six actuators (in a hexapod configuration) for each segment. These macroscopic motions are achieved with the same remarkable actuators ${ }^{26}$ (developed by Ball Aerospace) that ultimately are used to align and phase the PM segments to tens of nm precision.

Once the outer mirrors were deployed, initial checkout with the COCOA interferometers was performed and initial alignment moves of the PM segments executed. A "Consent to Proceed" meeting involving all stakeholders confirmed readiness of the payload for cooldown.

\subsubsection{Cooldown (days 9-40)}

The cooldown of the payload and GSE to the cryogenic temperatures was a slow and painstaking process. Numerous constraints on temperatures, rates of temperature change, and temperature gradients across structures had to be satisfied. In addition, temperature deltas among the various flight and GSE elements had to be monitored and properly maintained from a contamination standpoint, so that condensation of outgassing products did not occur onto critical optical systems. A key aspect of this process is the prevention of water ice deposition onto optical components. For the Science Instruments (SIs), mounted to a composite structure and surrounded by a thermal blanket assembly, this process involved the use of Contamination Control Heaters on the near-IR instruments to maintain their temperature at 165K (above water vapor condensing temperature at the relevant pressures) until the ISIM composite structure dropped below 140K. From there, the heater setpoints stepped the SIs down together (along with the cryo-cooler controlled MIRI) in 5K increments until they all were below water-releasing temperatures, at which point their cooling could proceed passively, while using heaters to satisfy any remaining constraints.

Structural gradients were monitored very closely, and the cooldown rate for the shroud could be adjusted as needed to stay within constraints. Though in general the thermal model did an excellent job of forecasting the cooldown ${ }^{16}$, there were significant departures in a few isolated regions, which led to the growth of temperature gradients threatening the general limits defined pre-test. In those cases, the structural/mechanical team did a superb job of analyzing the particular cases involved, establishing the safety of higher deltas, and allowing the cooldown to proceed.

PG runs were also very important for monitoring the safety of the cooldown, as the relative motions of hardware over this large physical scale and temperature range are substantial. When the chamber was evacuated, the hanging payload dropped by $>1 \mathrm{~cm}$ vs. the chamber floor, while during the cooldown to $\sim 40 \mathrm{~K}$, the long hanging rods shrank enough to raise the payload by nearly $5 \mathrm{~cm}$ with respect to floor-mounted hardware. Tracking of the PG-measured positions as well as the loads on the vibration isolators of the hanging configuration confirmed the absence of mechanical snags.

The six PM segments of the inner ring were deployed in the temperature range of deployment expected in flight. With all segments deployed, iterative alignment of the PM took place using the PG and COCOA systems - a moving target during cooldown, but good practice for using all of the analysis tools with 18 segments for the first time, after the twosegment Pathfinder experience. In the latter stages of the cooldown, two important Thermal Distortion/Figure Drift tests were executed ${ }^{27,28}$ - tracking the PM figure vs. temperature against model predictions; results are described below.

Between 120 and $100 \mathrm{~K}$, the near-IR instruments were brought online. They were used as soon as possible to dry-run optical test procedures, determining appropriate flux settings and pointing for the various light sources, thus reducing test overheads during the run-for-the-money execution of those tests during the cryo-stable portion of the test. Meanwhile, the GSE cryocooler brought the MIRI down to its 6K operating temperature. The ISIM reached its cryo-stable conditions earlier than the OTE; accordingly, the ISIM cryo-functional, known as its Comprehensive Performance Test, was begun in the latter stages of the cooldown. 


\subsubsection{Cryo-stable operations (days 41-75)}

When the payload reached its cryogenic target temperature range and minimal rate of change, cryo-stable operations began. During this phase, the cryo-functional tests of the OTE and ISIM elements were completed, along with mirror actuator fine range checks and calibrations ${ }^{29}$. The critical optical verifications and cross-check measurements were made: these included the fine alignment and phasing of the Primary Mirror, as well as the Half-Pass and Pass-and-a-Half test campaigns, including Wavefront Sensing and Control demonstrations. We elaborate on the details of those campaigns in the "Results" section below.

Closed-loop testing of the Fine Guidance Sensors with the Fine Steering Mirror was successfully completed ${ }^{30}$, along with guiding tests utilizing the Onboard Script System (OSS). Special instrument tests were executed. These included: spectroscopic searches for water ice contamination on the optics (none found), NIRISS pupil wheel alignment checks, NIRSpec light leak checks, an extensive suite of internal calibration lamp exposures in the NIRSpec for optical model verification (an important activity for this Multi-Object-Spectrograph, with wavelength calibrations needed for every combination of microshutter and dispersing element), and an exercise of the flight script for annealing the NIRSpec Microshutter Array (a contingency procedure in flight for freeing stuck microshutters). The MIRI Medium Resolution Spectrograph accomplished many calibration goals using a PAAH continuum source. Detector darks were acquired.

In parallel with these activities, thermal balance data was acquired for the individual science instruments, and measurements were taken to confirm the conductance of the SI heat straps and to measure the heat load onto the MIRI cryocooler. Toward the end of the cryo-stable period, an extended quiescent period for the OTE enabled the acquisition of a stable thermal balance for model validation. In parallel with that, operational testing of the ISIM continued, exercising flight scripts and flight-like operations in the Mission Operations and Day-in-the-Life tests with the OSS.

The above were all pre-planned activities; there was also a significant period of ad hoc investigation, before and after the OTE thermal balance, of instabilities that had been observed in the PM figure ${ }^{28}$. These investigations are also discussed in the "Results" section below.

The most dramatic occurrence of the OTIS CV test was the arrival, very near the beginning of the cryo-stable period, of Hurricane Harvey. This major storm dumped $~ 50$ inches of rain in the Houston area, including 22 inches at JSC over its first night. As can be imagined, this event posed major challenges for the test team, successfully overcome. The biggest risk was the loss of electrical power - though many facility systems were backed up with generators, an essential $\mathrm{He}$ cooling train was not. Recognizing the annual hurricane threat in the Houston area, extensive contingency planning ${ }^{31}$ had been done for this (and many other possible problems); a tricky warmup to LN2 temperatures, with the loss of weeks of test time, would have been required had power been lost.

Fortunately, power was not lost. Test operations continued with only a brief interruption. The biggest impacts were water leaks in the building that required moving or covering equipment and moving some stations for personnel, along with the challenges of keeping the test team safe in the face of extensive flooding in the area. Travel to and from JSC was impossible for the first 36 hours of the storm; people on-site had to remain on-site, sleeping in conference rooms. For the following several days, shift operations went to 12-hour shifts instead of 8-hour, to reduce the transportation exposure of the team going back and forth to their hotels, with all travel done in groups with high-clearance vehicles. Test operations returned to normal in about a week; JSC itself was officially closed for nominal operations for nine days. At the risk of embarrassing two of the authors of this paper, the rest of the authors would like to highlight OTIS Technical Lead Lee Feinberg and Lead Test Director Carl Reis for the superb leadership they exhibited in keeping both the invaluable personnel and the invaluable OTIS hardware safe through this event. The team was also very cognizant that, though the local topography allowed us to come through Harvey relatively unscathed, there were many in the Houston area less fortunate, including members of the JSC facilities team, who nonetheless continued to support the OTIS CV test with great dedication.

\subsubsection{Warmup (days 76-95)}

Toward the end of cryo-stable operations, a very thorough Consent to Proceed meeting was held, to document the successful accomplishment of the cryo-stable test goals, and to gain concurrence that the test was ready to proceed to warmup. With that concurrence in hand, warmup operations commenced. The warmup phase, like the cooldown, involved very careful monitoring and operations to ensure compliance with all structural and contamination constraints. One aspect of cooling the large He shroud to $20 \mathrm{~K}$ for two months is that the shroud became by far the largest cryo-pump in the system, condensing any outgassing products and the small but non-zero leak of outside atmosphere into the 
chamber. These condensed gases had to be released in a carefully controlled manner to avoid excess heat transfer among the various hardware elements, which could differ in temperature by as much as $270 \mathrm{~K}$.

Besides the basic goal of returning the hardware to room temperature safely, numerous other test goals were accomplished during the warmup period. These included a Thermal Distortion/Alignment Drift test, in which PG measurements tracked the positions of the PM, SM, and AOS over a $25 \mathrm{~K}$ temperature range, to confirm the adequacy of the Model Uncertainty Factor applied to distortions of the large structural hardware ${ }^{27,28}$. The motors and heaters for the major deployment systems (the Deployable Tower Assembly, the hinge drives for the Secondary Mirror Support System, and the latch drives for the PM wings) also underwent assorted tests during the warmup period.

Monitoring of COCOA interferometry and periodic alignment of the PM segments for the stability investigation continued through the warmup. As the temperatures approached room temperature, a warm OTE functional was run to confirm the health of that element after the long cryo cycle, and an analogous warm ISIM functional test was begun.

\subsubsection{Warm vacuum operations and backfill (days 96-100)}

In the final days of vacuum operations, the warm ISIM functional test was completed. The SM was stowed in its launch restraints (the PM segments were left deployed to support continued stability testing in ambient conditions after the vacuum test). Final vacuum investigations of the stability were performed, a final set of outgassing measurements was obtained, the PM Radius-of-Curvature actuators were set to their ambient positions, and the slow, careful backfill of the chamber was carried out. OTIS CV was complete, on the $21^{\text {st }}$ of October, 2017.

\subsection{General remarks on OTIS CV test execution}

In addition to detailing the specific test activities above, it is worthwhile to make a few general comments about the tactical and strategic execution of this mammoth undertaking.

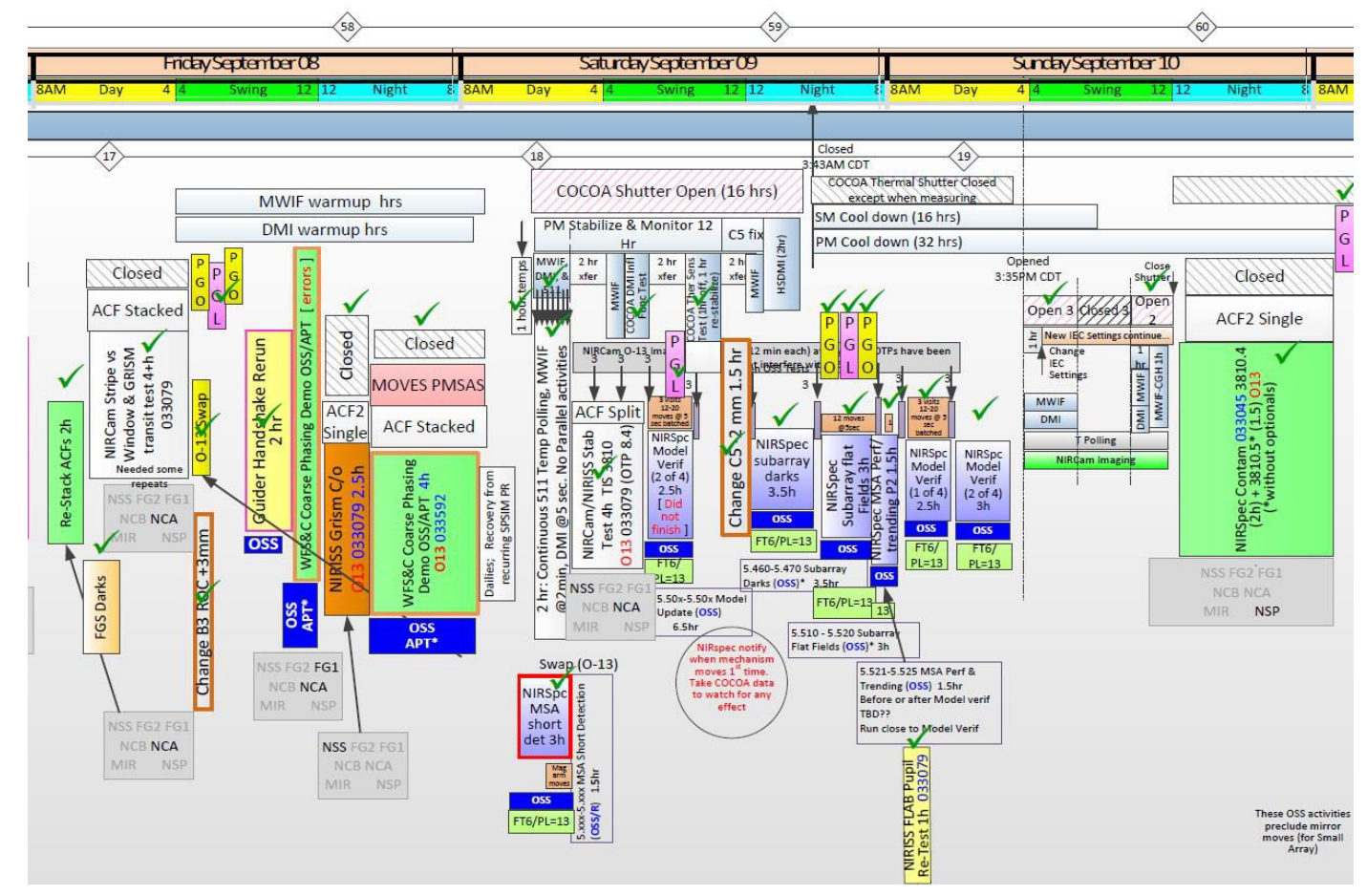

Figure 11. Activities performed in a mere three days of the OTIS CV test, as displayed in the elegant visual timeline tool developed by the OTIS CV Lead Test Planner, Mark Waldman. The intent here is not to explain this acronym-laden diagram in detail (though the diagram is fully legible when zoomed in online viewing), but simply to convey the myriad of activities that were going on during the test, many in parallel, and to give a sense of the utility of the visual planning tool for rearranging activities as required, by virtue of the fact that a great deal of critical information (SI usage, telemetry format requirements, COCOA shutter state, mirror configuration, etc.) was efficiently encoded in the display. 
Real-time execution of the test (round-the-clock, three shifts per day) was under the direction of an OTIS Test Director, with overall responsibility for the payload and the overall test flow, and a JSC Facilities Test Director, responsible for the vacuum operations for Chamber A. A Test Conductor guided the work through whichever specific test procedure was in process, directing the actions of a Test Operator, the single person on shift authorized to issue commands to the flight hardware. A substantial team operated and monitored thermal and optical GSE as well as each of the Science Instruments, archived incoming data, and performed quicklook analyses. Depending on test phase, 15-30 people were on shift at any given time in the JSC control rooms, augmented by numerous off-line team members providing test planning and data analysis support.

Stand-up shift handovers and shift reports distributed by each successive Test Director kept the test flow moving smoothly from shift to shift. More strategic decisions - response to problems, rearrangement of activities based on considerations of thermal status, data analysis results, etc - were under the purview of a Test Configuration Board, which met daily, chaired by the OTIS Technical Lead, Lee Feinberg. This core group, augmented by whatever discipline expertise was required, reviewed the overall progress of the test, discussed any issues that had been encountered, and decided on any changes that needed to be made to the pre-planned timeline. In making these changes, they were aided enormously by the visual timeline display tool (see Figure 11 above) and corresponding spreadsheets developed by Lead Test Planner, Mark Waldman, which were continuously updated by him and his deputy in this role, Tony Whitman.

Finally, major decisions involving hard-to-reverse changes in test configuration (e.g. decisions to start cooldown or warmup) were agreed upon in formal Consent to Proceed meetings with OTIS management and all of the major stakeholders of the project.

\section{OTIS CRYO-VACUUM TEST RESULTS}

\subsection{Functional test results}

Besides the specific functional tests conducted for both the OTE and the ISIM, under both ambient-vacuum and cryovacuum conditions, the telescope and instruments were of course utilized extensively to accomplish the optical and operational testing goals of OTIS CV. All of these activities confirmed that the entire flight payload had come through its environmental test exposure in excellent health.

The OTE, experiencing its first full-up cryo-test, showed completely nominal performance for its large number of essential mirror actuators: $132=18$ PM segments x 7 [6 (hexapod) +1 (Radius-of-Curvature, RoC) actuators)] + 6 SM (its hexapod, no RoC). The corresponding positional readouts for those actuators also performed as expected (three of the large number of position sensors had issues, but these had been evident since the OTE was first integrated, accepted when first recognized because adequate redundancy remains). Similarly, the motors, readouts, and heaters for the major deployable elements performed completely nominally.

On the ISIM side, the instruments also continued to show excellent health and nominal performance. One particularly important aspect of the NIRSpec testing was the first opportunity to examine the state of the Microshutter Array (MSA, operable only at cryo) after its OTIS proto-flight level acoustic exposure, to which the arrays are known to be sensitive; the MSA checks demonstrated that no significant degradation of the array had occurred from that exposure ${ }^{32}$.

One significant SI change was noted, generating what is known as a Problem Failure Report (PFR): a significant image shift was noted in the Shortwave $(0.6-2.5 \mu \mathrm{m})$ Channel of one of the two redundant NIRCam modules. The data acquired to troubleshoot this issue during OTIS CV and the subsequent analyses by the PFR Board have determined that this shift has resulted from one loose bond in the mount of a fold-mirror optic in the relevant channel (a bond that retrospective analysis indicates has been loose since before ISIM-level testing). The Board has determined that the remaining bonds are structurally sufficient to be safe to fly, and the plausible launch shifts for the optic are of minimal scientific consequence, so no repair is required. One other SI PFR was opened, for communication dropouts to one of the MIRI electronics boxes. Subsequent investigations have shown that the root cause of this behavior was very likely poor electrical contact in relays and connectors of the electrical GSE, with no implications or harm to the flight hardware.

\subsection{Optical test results}

The over-arching result of OTIS CV optical testing is that all of the optical test objectives were met. All >30 tests in the prioritized optical test matrix (of requirement verifications, critical cross-checks, and demonstrations) were successfully executed, with excellent results. Many results are reported elsewhere in the proceedings of this conference; here, we 
highlight key optical test results and point where appropriate to those more detailed presentations. Lightsey et al. $(2018)^{33}$ also provide quantitative performance summaries for tests whose results are discussed more qualitatively below.

\subsubsection{Photogrammetry performance and results}

The four PG cameras on their rotating booms performed superbly in OTIS CV, through dozens of full rotational runs, involving many thousands of lamp flashes. Sample images are shown in Figure 12.
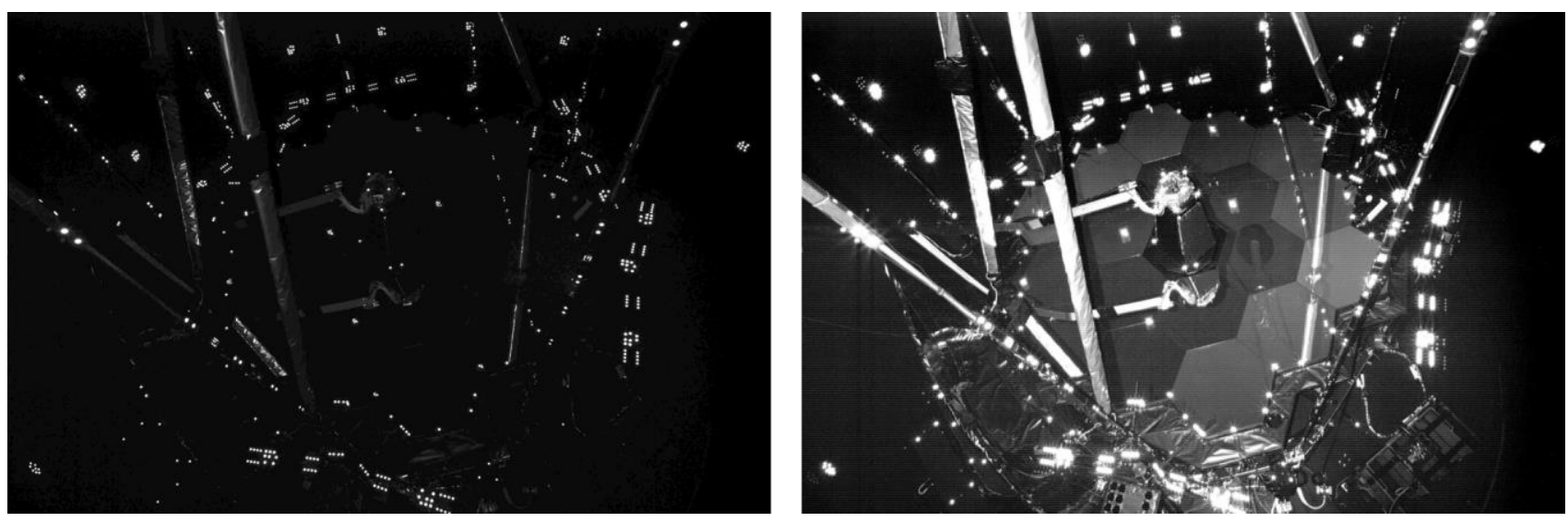

Figure 12. Left) Representative single flash image from a PG run, from one of the cameras, showing the returns from the payload- and GSE-mounted optical targets. Returns are seen, for example, from targets mounted to the outer edges of the PM segments, as well as the scale bars used for calibration of the images. Right) A composite of many images taken from the same PG position, bringing out details of the payload under test. In addition to the PM segments, visible items include the hanging rods, the AOS, and the arm supporting the fiber feed to the AOS Source Plate Assembly.

As demonstrated previously in Pathfinder testing ${ }^{21}$, the precision and accuracy of the PG analyses was remarkable. OTIS CV runs demonstrated two-sigma repeatability at the levels of 10's of $\mu \mathrm{m}$, with absolute accuracies of $\sim 100 \mu \mathrm{m}$ (over a payload volume of $\sim 7 \mathrm{~m} \times 7 \mathrm{~m} \times 10 \mathrm{~m}$ ), well satisfying the requirements for PG-guided alignments.

PG data guided the alignment of the PM segments (in concert with COCOA interferometry), including their absolute positioning with respect to the AOS. As events transpired in the OTIS test, final alignment and phasing of the PM segments was made with the PM decentered with respect to the AOS (as determined by subsequent PG analysis) by $0.45 \mathrm{~mm}$ radially, more than intended, due to a temporary error in analyzing COCOA data. Once this offset (and the source of the error) was recognized, the Test Configuration Board discussed whether it was necessary to iterate further and rephase the telescope after correcting that offset. It was concluded that it was not required; the origin of the offset was understood, it had negligible wavefront or pupil implications, and could be readily corrected for in assessing the actuator range budget and SM alignment. And the hurricane was coming. See Hadaway et al. (2018) ${ }^{14}$ for additional details. PG data similarly guided the rough alignment of the SM, before that process was ultimately completed with Pass-and-a-Half observations.

\subsubsection{Mirror alignment and phasing results (COCOA interferometry)}

Many hundreds of Multi-Wave Interferometer (MWIF) runs were made over the course of OTIS CV testing, each consisting of tens to hundreds of individual exposures. The alignment and phasing process involved correcting PM segment piston errors progressively via a step-down process utilizing progressively shorter "synthetic wavelengths" obtained from MWIF images at closely spaced wavelengths. The PM segments were then aligned in tilt, radial decenter, clocking, and radius of curvature to minimize total PM wavefront error (WFE). Sample interferometer images of the phased mirror are shown in Figure 13. PM WFE meeting test requirements was achieved (e.g. 118nm Peak-to-Valley piston errors, $34 \mathrm{~nm}$ rms, vs. a $150 \mathrm{~nm}$ P-V requirement; segment tilts <83nrad). Comparison of the final observed surface shape for the PM segments was also in excellent agreement with model predictions for these light-weight beryllium segments on their hexapod mounts in a 1-g field (Figure 14). Again see Hadaway et al. $(2018)^{14}$ for details.

The COCOA systems and the Absolute Distance Mirror Assembly, mounted behind an edge of the PM, were also used, along with the segment radius-of-curvature actuators to set the PM radius of curvature to its nominal value within requirements, and to measure its conic constant, also within requirements. See Cosentino et al. (2018) ${ }^{34}$ for details. 

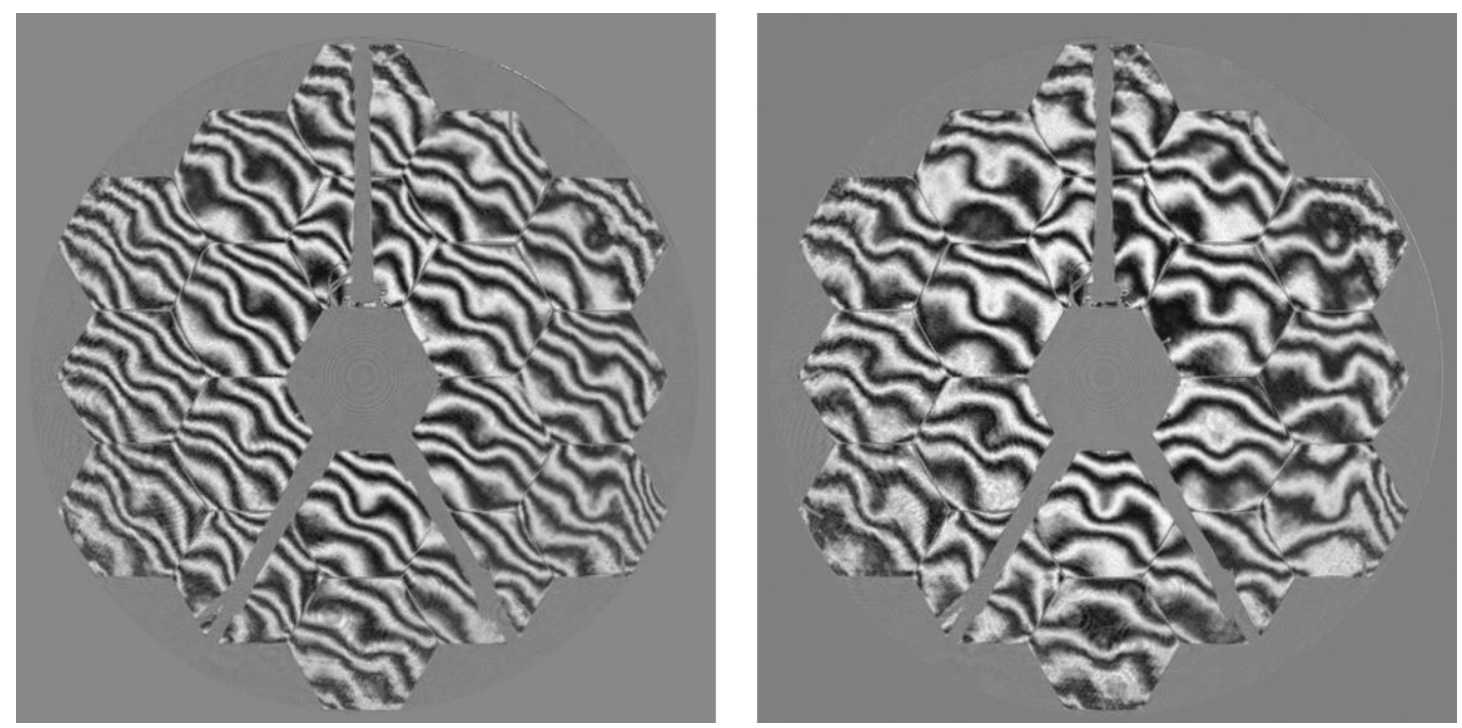

Figure 13. Fringe images from the COCOA interferometer for the aligned and phased JWST Primary Mirror. Blockage by the Secondary Mirror support tripod is shown. Note the continuity of the fringes across segments; the higher fringe density at left simply reflects greater tilt of the COCOA interferometer with respect to the PM. The waviness of the fringes is a consequence of the gravity effects on the light-weighted mirror segments, as described below.
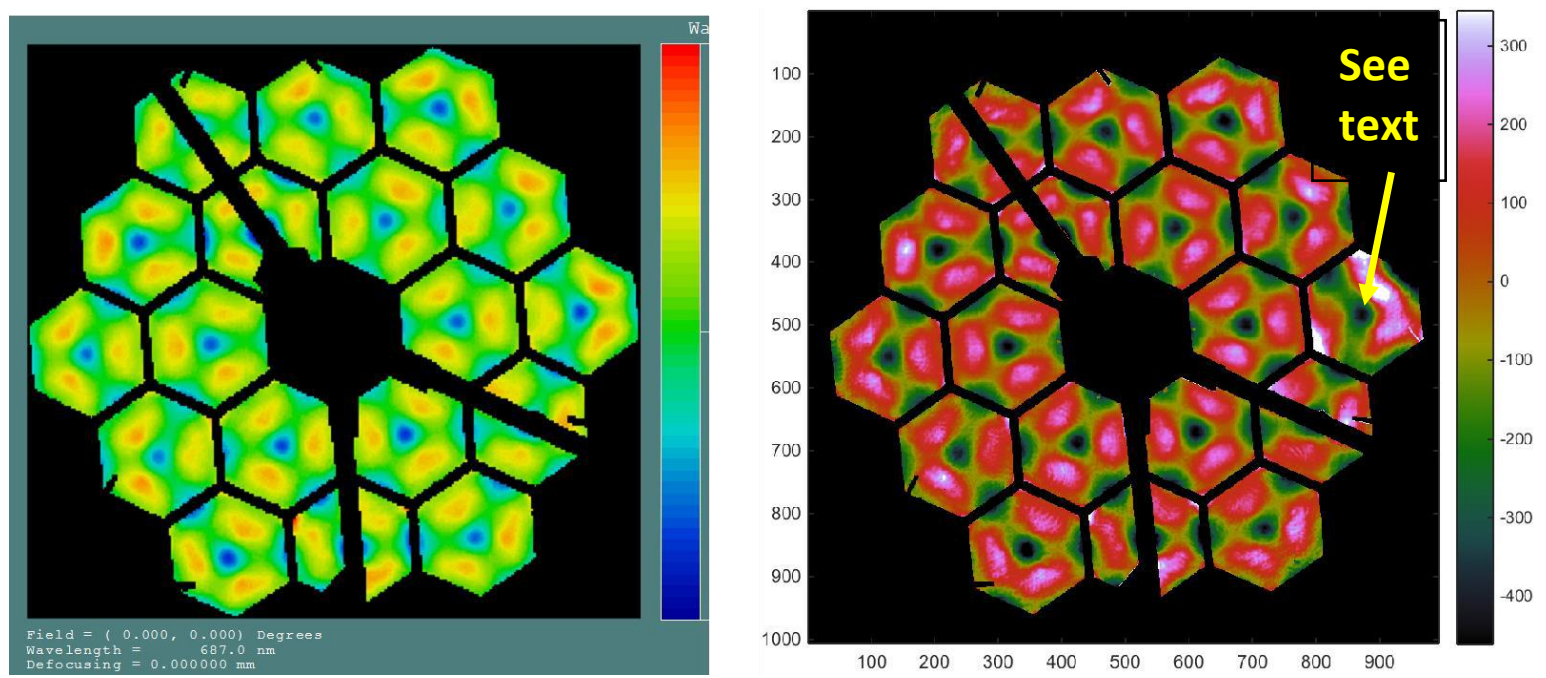

Figure 14. Left) Modeled wavefront errors for the PM segments in the 1-g field. Right) The corresponding measured results, showing excellent agreement for the light-weighted segments on their hexapod mounts. The theoretical minimum was $158 \mathrm{~nm}$, while the measured value, with its measurements errors, was $182 \mathrm{~nm}$, including the outlier segment indicated at the far right. The distortion seen for that segment resulted from a non-flight-relevant interference (see text).

The segment wavefront results in Figure 14 provide an excellent example of an optical cross-check in OTIS CV. While the WFE precisions in the OTIS CV test were not as fine as in the segment-level cryo-testing that was used to verify their figures vs. their $25 \mathrm{~nm}$ rms surface requirement, the accuracy was sufficient to show that there was something wrong with the B3 segment in the end-to-end OTIS test, as shown by its significant distortion in the right-hand panel of Figure 14. Fortunately, it was realized that the likely cause was an interference between the thermal blanket closeouts around the PM and the non-flight photogrammetry target mounted to the edge of that B3 segment, when the segment was in its fully deployed state. An experiment was performed to back off that segment in piston by $2 \mathrm{~mm}$ to reduce the interference. A dramatic reduction in distortion was achieved. Subsequent modeling showed superb agreement in shape with predictions for the change due to a force input at the PG target location ${ }^{28}$. PM interferometry was thus of sufficient accuracy to serve as a highly useful cross-check - flagging a potential figure problem with a PM segment - but it was fortunately resolved as a non-flight-relevant issue. 


\subsubsection{Half-Pass alignment results}

Half-Pass (HP) testing was fully successful in confirming the critical alignment between the AOS and the ISIM and its instruments. This was a particularly high-priority requirement verification because that alignment is not adjustable in flight. The near-IR instruments have focus mechanisms, but MIRI does not, and only the NIRCam has a pupil alignment capability. The other SIs needed to be properly aligned with respect to the Fine Steering Mirror and telescope exit pupils a priori, based on prior metrology and accurate installation of the ISIM onto the mirror backplane. HP testing with the inward sources, which included measurements in all instrument channels, confirmed that the focus, boresight, and pupil alignments were indeed within requirements.

Figure 15. Sample images from the Half-Pass test campaign. As discussed in Section 2.1, the images from the Cassegrain focus of the JWST three-mirror anistigmat are highly aberrated. However, they are perfectly modelable, and the analysis of images such as those at the right, from all instrument channels, permitted verification of the ISIM alignment to the AOS to within flight requirements.

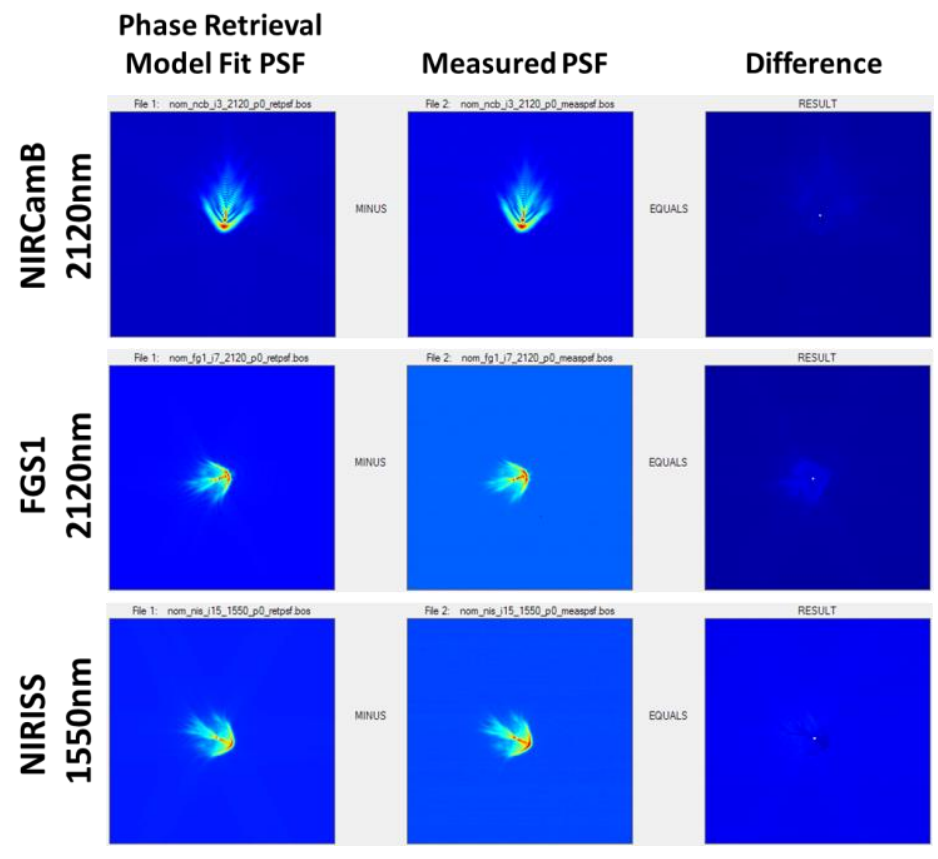

\subsubsection{Pass-and-a-Half alignment results}

Pass-and-a-Half (PAAH) observations using the outward-facing sources of the AOS Source Plate Assembly were used, among other objectives, to align the Secondary Mirror.
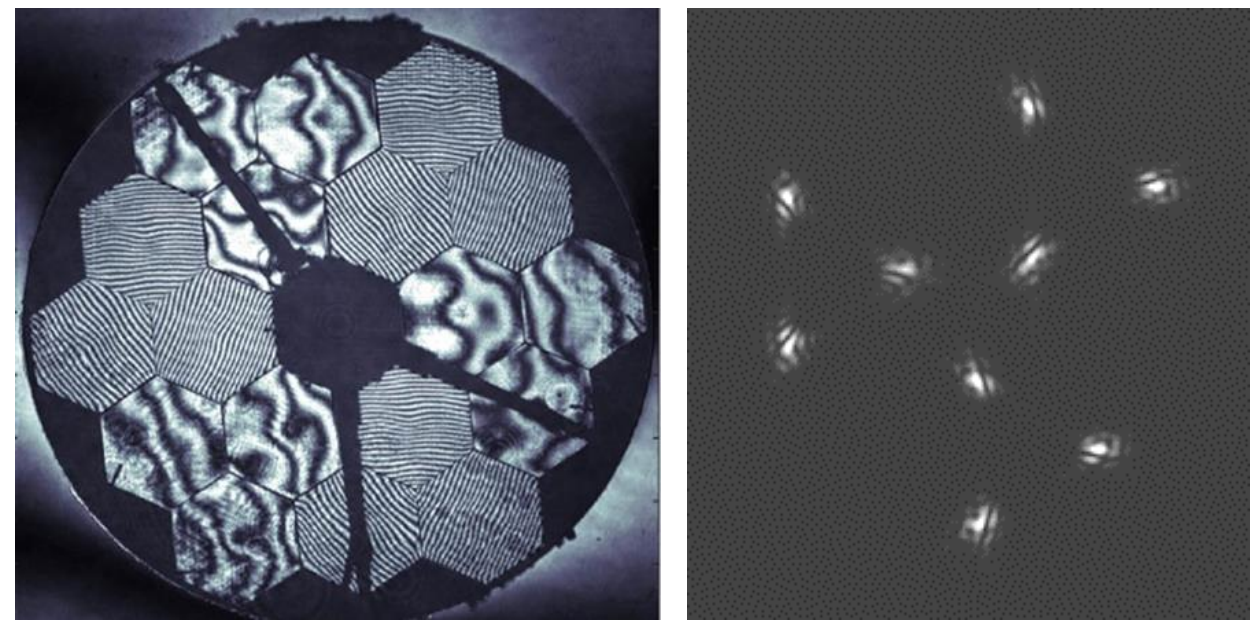

Figure 16. Many PAAH alignment measurements were made in the Hartmann configuration, achieved by tilting the three PM segments beneath each Auto-Collimating Flat outward (as indicated in the interferogram at left), and then tilting the ACFs themselves to separate the three resulting image triads, shown at the right in a sample NIRCam image. 
The Hartmann configuration for SM alignment had been decided upon based on OGSE2 Pathfinder testing, as a means of mitigating the chamber vibration seen in that dry run $^{35}$. Phase retrieval analyses are very sensitive to the image blur produced by such vibration, but in Hartmann analyses, the results are derived from spot centroids from exposures long enough to average over the vibration and thus produce stable results. Analyses of SM focus sweeps and measurements of SM influence functions (the responses of the Hartmann triads to SM tip/tilt and decenter motions) enabled crosschecking the alignment of the SM to the required accuracies. A clever technique of inverting the tilts of the PM segment triplets eliminated some systematics (e.g. plate scale errors) from the analyses, improving the accuracy of the results. OTE field tilt was also characterized and was satisfactory. See Coyle et al. $(2018)^{36}$ for details of the PAAH alignment process.

\subsubsection{PAAH wavefront sensing $\&$ control results}

PAAH testing was also used for important functionality checks of the NIRCam elements that support the observatory's wavefront sensing \& control procedures and for exercising the relevant scripts and data pipeline processing. Use of NIRCam's Dispersed Hartmann Sensor (DHS) masks to perform coarse phasing measurements is depicted in Figure 17.
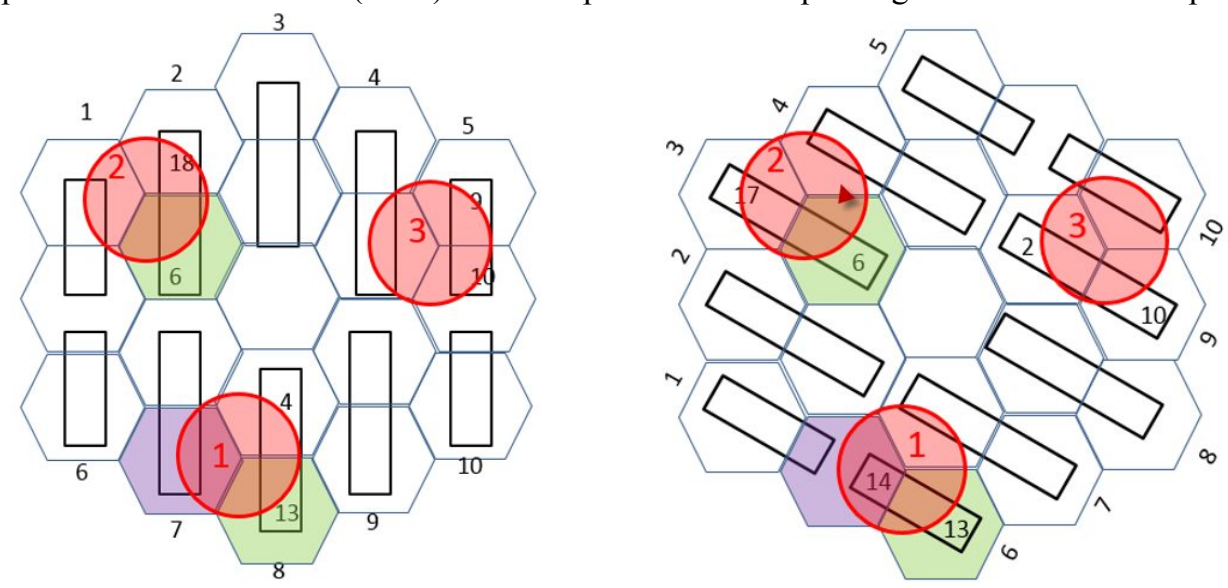

Figure 17. Schematic diagrams of the pair of DHS masks located in the NIRCam Shortwave Channel pupil wheel ${ }^{37}$. Each mask element straddles two PM segments, and relative piston between the two segments can be derived from the resulting spectral fringes produced using the NIRCam grism. In flight, the two 60-degree-different orientations permit relative measurements for all 18 PM segments. For OTIS CV, the red circles indicate the regions where the ACF returns provide illumination for use in piston-measurement demonstrations.

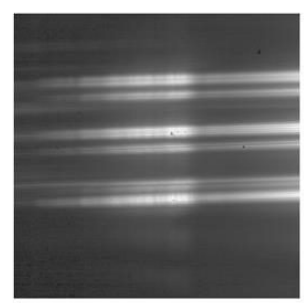

Before piston

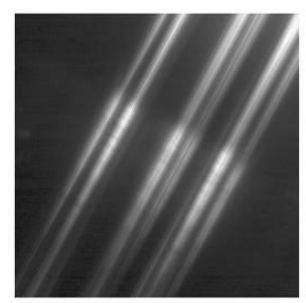

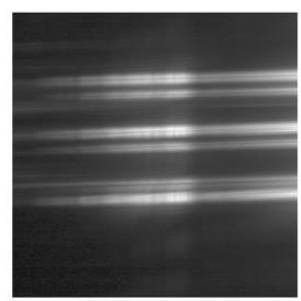

After piston

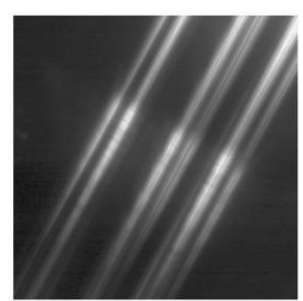

Figure 18. Sample images from the coarse phasing demonstration using the two orientations of NIRCam DHS masks. Missing spectral stripes correspond to DHS apertures that are not illuminated through the OTIS CV PAAH setup (Figure 17).

The images at left show the global pattern for each orientation. The characteristic spectral fringe pattern from which the relative piston of two segments is inferred is shown in the extracted image below. See Acton et al. $(2018)^{37}$ for details.

\section{Example of an extracted fringe}

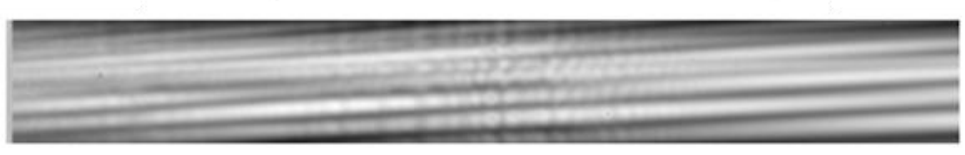

The wavefront sensing \& control hardware functionality checks and procedural demonstrations (including the use of the full ground system infrastructure at the JWST Mission Operations Center at STScI to process the data) went very well. Details can be found in Acton et al. $(2018)^{37}$ and Lajoie et al. $(2018)^{38}$, in this volume. 


\subsubsection{Thermal distortion results}

As noted in Section 3.2, several periods of thermal transition during the OTIS CV test were used to provide valuable cross-checks of the distortion of the telescope's optical system caused by changes in temperature. The temperature ranges sampled, during both cooldown and warmup, represented significant overdrive (larger temperature deltas) than anticipated in flight operations, in order to get the most sensitivity out of the accuracies of the available measurements. The goal was to confirm the adequacy of the Model Uncertainty Factor (MUF) that is applied when predicting thermallyinduced distortions in flight.

During cooldown, two different time periods - temperature change intervals - were used to characterize figure distortion of the primary mirror (caused principally by changes in the shape of the composite backplane). The second figure drift test was compromised by an instability described in Section 4.2.7 below, which has since been shown not to be flightrelevant. However, the cleaner, first figure drift test demonstrated excellent agreement between model predictions of the wavefront change and the measured results: extremely close when parameterized simply by the change in rms wavefront (Figure 19), and still well within the MUF when evaluated as the rms difference in the observed shapes of the wavefront.

Alignment drift tests during both cooldown and warmup used the PG system to track the relative positions of the PM, SM, and AOS over even larger temperature overdrive ranges. These tests also supported the adequacy of the MUF used to predict flight performance. See Howard et al. (2018) $)^{27}$ and Atkinson et al. (2018) ${ }^{28}$ for details of the thermal distortion test program.

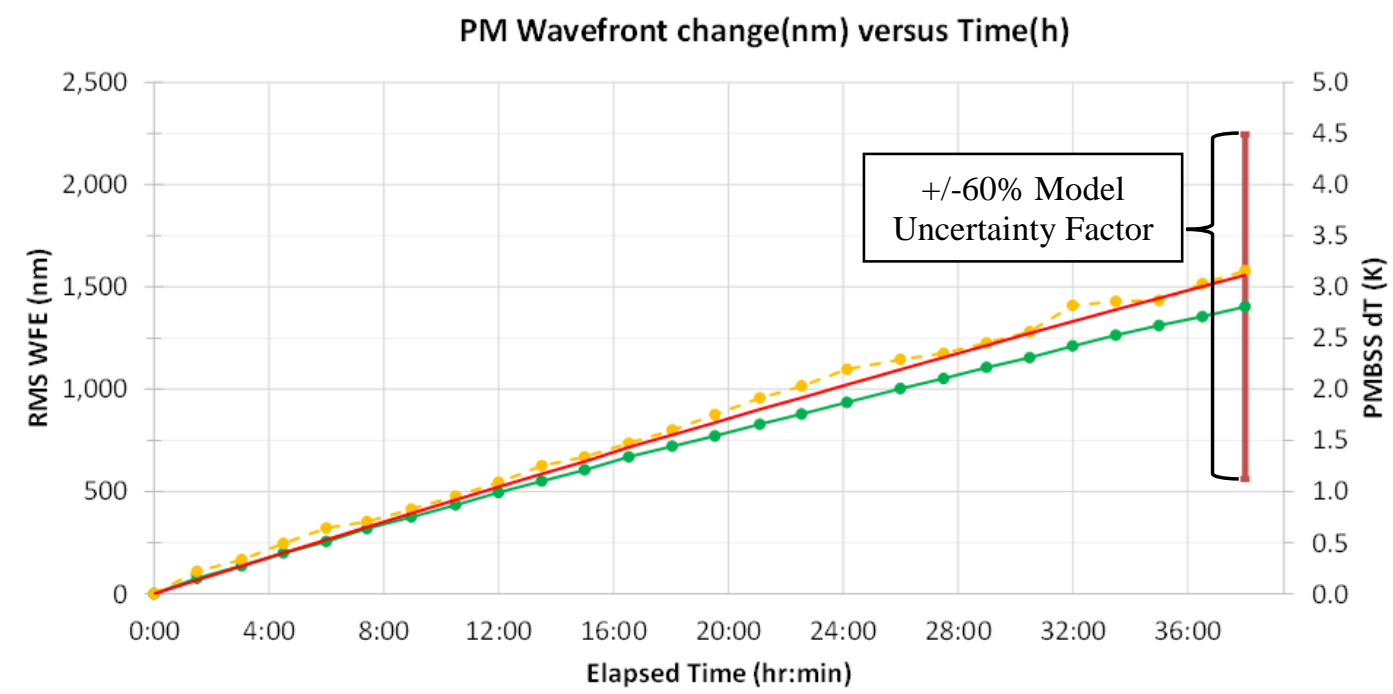

Figure 19. Results of the first figure drift test performed during cooldown. The plot shows the predicted rms WFE change in green and the measured values in orange. The difference between measurements and prediction was well within the Model Uncertainty Factor that the JWST program uses in predicting such thermal distortion effects in flight ${ }^{27,28}$.

\subsubsection{Primary mirror instabilities}

The most disquieting phenomena observed in the OTIS CV test program were various forms of figure instability observed for the primary mirror. These included periodic oscillations over tens of minutes in wavefront shape, with an amplitude of $>100 \mathrm{~nm}$ rms, a $\sim 360 \mathrm{~nm}$ systematic rise in rms WFE over the course of hours in response to opening of the COCOA shutter, and discrete "tilt events" (sudden jumps in the tilt of individual PM segments between interferometry runs). These behaviors inspired significant ad hoc experimentation, under the direction of the PFR Board convened during the $\mathrm{CV}$ test, in order to characterize the behaviors and understand their source. Details are presented by Atkinson et al. $(2018)^{28}$, in this volume; we present a few highlights here.

The periodic wavefront oscillations were traced during the $\mathrm{CV}$ test to originate in the cycling of heaters on the radiator panels of the ISIM Electronics Compartment. The expansion/contraction cycles of the radiator panels induced by the bang-bang cycling of those heaters was found to couple in an unexpectedly strong way to distortions of the PM 
backplane and thus of the mirror figure. Figure 20 illustrates the results of an experiment to change the width of the heater control deadband, which resulted in a reduction in the amplitude and shortening of the period of the oscillations.

Figure 20. The PM wavefront oscillation period and amplitude changed with the IEC heaters' temperature control deadband. (X-axis in minutes; Y-axis in $\mathrm{nm}$ rms.)

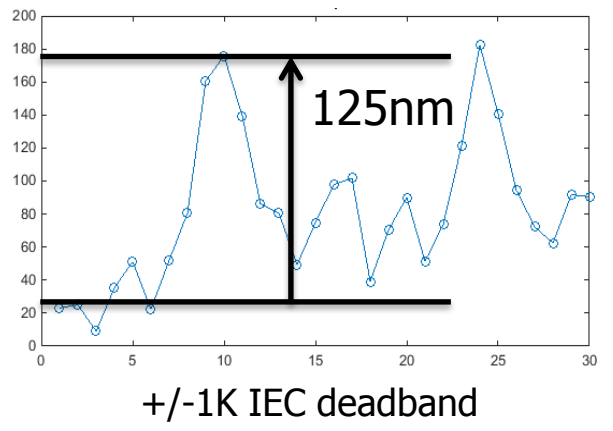

Extensive experimentation was carried out to characterize the IEC-induced behavior, during the cryo-stable test period, during warmup, and in subsequent testing under ambient conditions (possible because the PM wavefront changes are measured with the COCOA interferometer, not with the cryogenic science instruments). The ambient experiments proved (by changing the stiffness of the support of the IEC) that the large amplitude was driven by the much-stiffer-thanflight flexures that supported the IEC in test. Model predictions for the behavior with the lower stiffness of the flight flexures predict very small oscillations, well under stability requirements, so this issue is considered fully resolved.

The second unexpected phenomenon involved a wavefront change of $360 \mathrm{~nm}$ rms seen when the COCOA shutter was opened after an extended period closed (Figure 21). This configuration change represents a large change in radiant power incident on the telescope (and, more importantly, it was found) onto the frill and PM segment blanket closeouts. The frill is the black kapton assembly surrounding the periphery of the primary mirror, as can be seen in Figure 2; it serves the purpose of blocking stray light from behind the telescope, important for the open JWST telescope architecture.

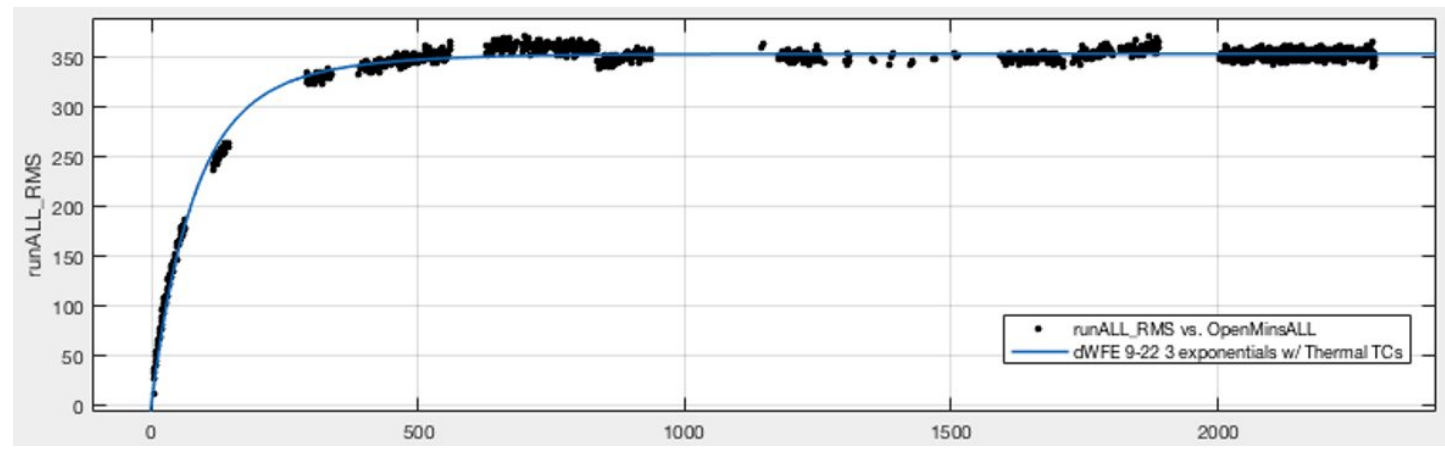

Figure 21. The change in rms wavefront (in $\mathrm{nm}$ ) vs. time (in minutes) after opening of the COCOA shutter. Please note that this figure represents the results of updated analysis vs. the corresponding figure in Atkinson et al. $(2018)^{28}$.

Inspection of PG images from the CV test and examination of the frill and blanket closeouts after the test showed that the primary cause of the distortion was insufficient slack in the frill and blanket assemblies, such that when the Kapton assemblies shrank during cooldown, they exerted changing forces and torques on the mirror backplane in response to temperature changes. Scaling to the small temperature changes expected in flight scientific operations, the expected PM figure effects are reduced by an order of magnitude. In addition, small modifications to the implementation of the frill have been made in a number of locations to restore the slack intended in the design, which had been compromised by some installation processes. Modifications to the PM closeout blankets for the same purpose are currently being evaluated. (The trade is between disturbing tested hardware vs. the amplitude of the predicted flight effects.) The upshot is that manageable WFE variations are expected due to this phenomenon in flight.

Mining of the vast OTIS CV data set is still ongoing with respect to tilt events, but it is clear that this phenomenon was most noticeable during cooldown, as the structures adjusted to the significant changes in stress. During the cryo-stable operations, few such events were observed, and their relevance to nominal flight operations is still being assessed. 


\subsection{Thermal test results}

Thermal testing of the OTIS payload also very successfully accomplished its goals (see Yang et al. [2018 $]^{16}$ ). The biggest success was in safely cooling and warming the hardware and maintaining the highly non-equilibrium cryo-stable state for the many weeks in between. This was accomplished while satisfying the temperature deltas required to keep critical optical surfaces free of condensed contamination as well.

All flight hardware was successfully cooled to its flight operating temperature range. A sufficiently stable thermal balance was achieved for model validation, both for the OTIS as a whole, and for the individual science instruments within the ISIM. Health of heaters and temperature sensors was confirmed, and the workmanship of the critical instrument heat straps was confirmed - unchanged (within uncertainties) conductance was observed for those items after the OTIS environmental test program.

There was one very localized discrepancy in the observed temperatures vs. model predictions that was large enough to generate a Problem Failure Report. A small portion of the mirror backplane, near but not touching the warm IEC, ran $22 \mathrm{~K}$ above model predictions, at $\sim 92 \mathrm{~K}$ vs. $70 \mathrm{~K}$ expected. Model adjustments since the test have since fully explained the deviation. The adjustments required were, principally, correcting the model for a bracket inadvertently left floating, adding the high emissivity of some dry-film-lubricated surfaces, and using a conductivity for the composite backplane material that better fits the broad temperature distributions observed for that structure. The effects of these adjustments were so high only in this region, because it is a place that $270 \mathrm{~K}$ IEC components are very close to $70 \mathrm{~K}$ components.

Finally, very useful measurements were made with respect to the MIRI cryocooler. Though the bulk of the flight cryocooler has undergone an independent test program and was not present for this test (a GSE cooler was used for OTIS CV testing), the flight cold head mounted to MIRI itself, and all of the flight "plumbing" and field joints down to the spacecraft core region were present. This allowed good measurements to be made of the heat loads in various regions that the cryocooler will be required to lift; the measured loads met requirements. Successful measurements were also made with respect to the allowable leak rate of the helium cooling gas from the cooler system. It had been thought that meaningful measurements would not be feasible in the face of the significant He background from chamber and $\mathrm{He}$ cooling shroud leaks. However, the cooler team devised an elegant experiment in which they partially filled the cooler with ${ }^{3} \mathrm{He}$ instead of ${ }^{4} \mathrm{He}$. Tuning the chamber leak detector to ${ }^{3} \mathrm{He}$, where the chamber background was negligible, they confirmed that the leak rate of the portions of the cooler system present was below requirements.

\subsection{Operational test results}

The operational aspects of the OTIS payload were extensively and productively exercised during the CV test as well. This included the extensive use of flight scripts for instrument and OTE operations (such as mirror moves) and for flight demonstrations. Of particular importance was the closed-loop testing of the Fine Guidance Sensors with the Fine Steering Mirror, described in detail in this volume by Vila Costas et al. $(2018)^{30}$. This was the only cryo-test in which the FGS and FSM could be used together in a closed-loop way. Functional, polarity, alignment, and timing checks were conducted, all successfully, despite the fact that the Guiders had to centroid on the highly aberrated Half-Pass images.

\section{Centroid Spiral Plot (Test Data and Ideal Sim Pattern)}
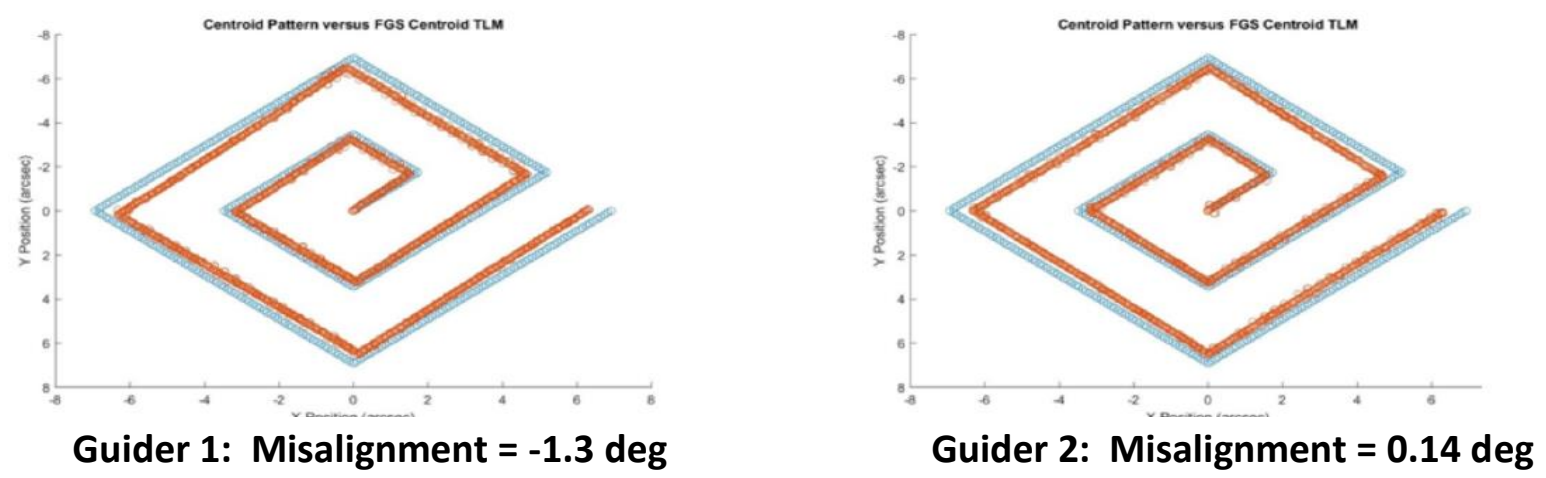

Figure 22. Spiral test patterns for the FSM, as imaged by the Guiders (commanded [blue] vs. observed [red] positions) ${ }^{29}$. Rotational alignment of the Guider readouts is within requirements, and the small scale mismatch will be tweaked with an adjustable parameter in the FSM's tip/tilt control. 
Wavefront sensing \& control demonstrations have already been described above (Section 4.2.5). In addition substantial runs of flight OSS scripts were made in parallel with the OTE thermal balance, in the Mission Operations and Day-inthe-Life tests. The latter emulated flight-like sequences of operations as closely as possible in the test environment.

\section{SUMMARY}

We have described the execution of a challenging, remarkably successful cryo-vacuum test campaign of the OTIS: the combined Optical Telescope Element and Integrated Science Instrument Module for the James Webb Space Telescope. Over a 100-day, round-the-clock test campaign, the OTIS underwent a battery of functional, optical, thermal, and operational tests. The test demonstrated the functional health and high performance of the OTIS hardware. Alignment of the adjustable elements of the telescope was demonstrated within requirements, with residual actuator range sufficient to meet required allocations for measurement uncertainties and ground-to-flight changes. (This telescope must be aligned again when it is deployed in space; the OTIS CV test demonstrated that it can be aligned within flight performance requirements.)

All major objectives of the test program were accomplished, including the full prioritized matrix of optical requirement verifications, cross-checks, and demonstrations (see Lightsey et al. [2018] ${ }^{33}$ for additional quantitative details). The expectation, based on OTIS testing, is that the JWST payload will meet its specifications and deliver the revolutionary science it has been designed for (see e.g. McElwain [2018 $]^{1}$ for sensitivity projections). In closing, Figure 23 presents the beautiful OTIS payload in the JSC cleanroom after its emergence from the vacuum chamber. Since then, the OTIS has been shipped to Northrop Grumman Aerospace Systems in California. It has been successfully functionally tested there, post-shipment, and awaits integration with the Spacecraft Element (warm spacecraft and sunshield) to complete the JWST Observatory.

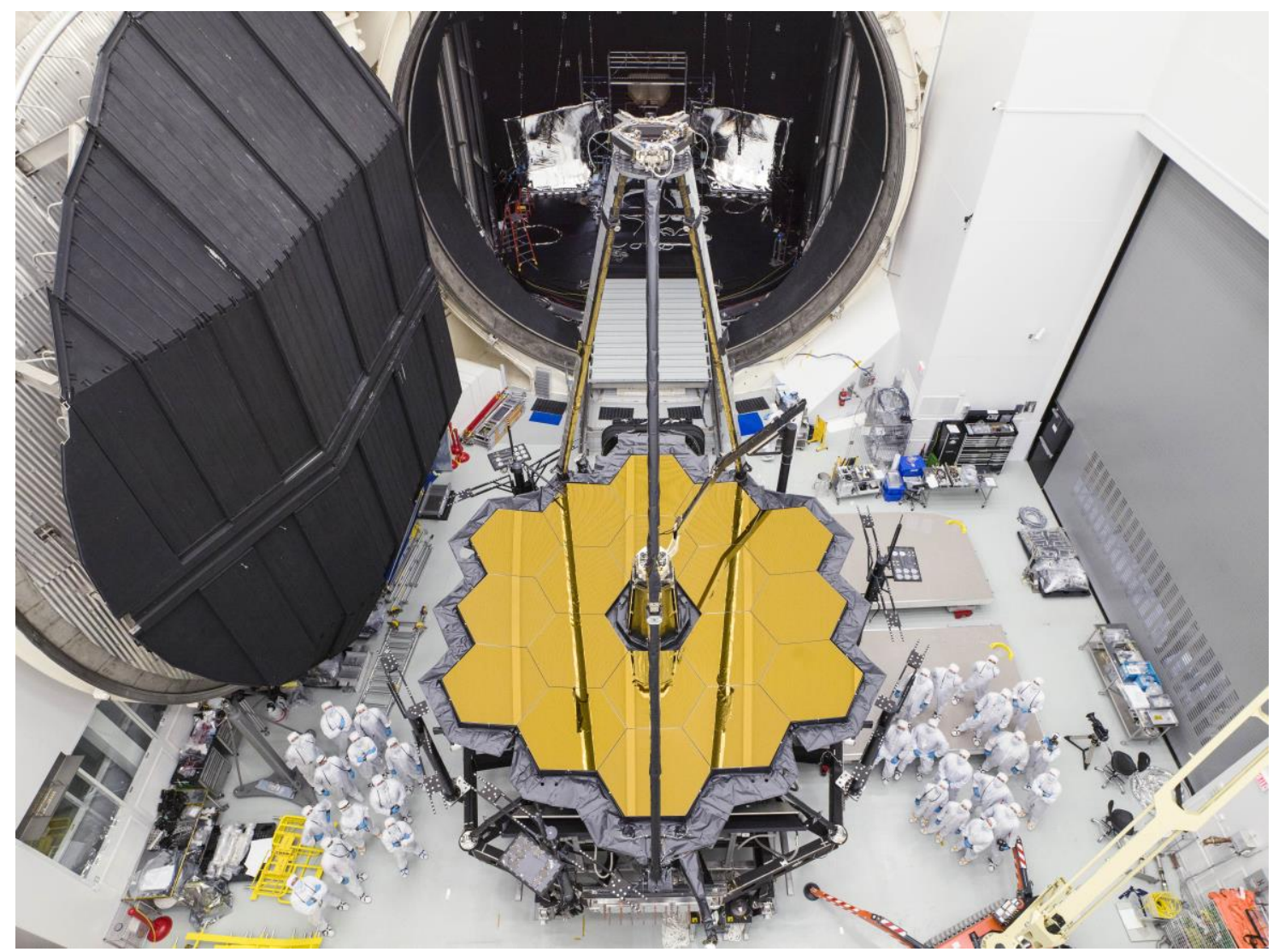

Figure 23. The OTIS, in the cleanroom outside of JSC's historic Chamber A, after its successful cryo-vacuum test campaign. 


\section{ACKNOWLEDGMENTS}

The JWST program is a collaboration of the National Aeronautics and Space Administration, the European Space Agency, and the Canadian Space Agency. The authors gratefully acknowledge all of the incredibly dedicated members of the OTIS test team and the JSC facilities team for their expert and unflagging support of the grueling, but highly successful, cryo-vacuum test campaign described above.

\section{REFERENCES}

[1] McElwain, M. W., Niedner, M. B., Bowers, C. W., Kimble, R. A., Smith, E. C., Clampin, M., "The James Webb Space Telescope" observatory status and preparations for launch," Proc. SPIE 10698, in press (2018).

[2] Atkinson, C., Texter, S., Keski-Kuha, R., Feinberg, L., "Status of the JWST optical telescope element," Proc. SPIE 9904, 990403 (2016).

[3] Rieke, M., Kelly, D. and Horner, S., “Overview of James Webb Space Telescope and NIRCam's Role," Proc. SPIE 5904, 1-8 (2005).

[4] Beichman, C. A., Rieke, M., Eisenstein, D., Greene, T. P., Krist, J., McCarthy, D., Meyer, M., Stansberry, J., "Science opportunities with the near-IR camera (NIRCam) on the James Webb Space Telescope," Proc. SPIE 8442, $84422 \mathrm{~N}(2012)$.

[5] Bagnasco, G. et al., "Overview of the near-infrared spectrograph (NIRSpec) instrument on-board the James Webb Space Telescope (JWST)," Proc. SPIE 6692, 66920M (2007).

[6] Birkmann, S. M. et al., "The JWST/NIRSpec instrument: update on status and performances," Proc. SPIE 9904, 99040B (2016).

[7] Rieke, G. H. et al., "The Mid-Infrared Instrument for the James Webb Space Telescope, I: Introduction," PASP Vol. 127, Issue 953, 584-594 (2015).

[8] Wright, G. S. et al., "The Mid-Infrared Instrument for the James Webb Space Telescope, II: Design and Build," PASP Vol. 127, Issue 953, 595-611 (2015).

[9] Doyon, R. et al., "The JWST Fine Guidance Sensor (FGS) and Near-Infrared Imager and Slitless Spectrograph (NIRISS)," Proc. SPIE 8442, 84422R (2012).

[10] Maszkiewicz, M., Saad, K., Rowlands, N., Doyon, R., Hutchings, J. B., "JWST Fine Guidance Sensor and NearInfrared Imager and Slitless Spectrograph,” Optical Payloads for Space Missions (ed S.-E. Qian), ch35, (2015).

[11] Kimble, R. A. et al., "Cryo-vacuum testing of the JWST Integrated Science Instrument Module," Proc. SPIE 9904, 990408 (2016).

[12] Saif, B., et al., "JWST center of curvature test method and results," Proc. SPIE 10401, 104010T (2017).

[13] Wells, C., Olczak, G., Merle, C., Dey, T., Waldman, M., Whitman, T., Wick, E., Peer, A., "The center of curvature optical assembly for the JWST primary mirror cryogenic optical test: optical verification," Proc. SPIE 7790, 779003 (2010).

[14] Hadaway, J. H., et al., "Performance of the center-of-curvature optical assembly during cryogenic testing of the James Webb Space Telescope,” Proc. SPIE 10698, in press (2018).

[15] Knight, J. S., Gallagher, B., Frazier, D., Whitman, T. L., Feinberg, L. D., Jhabvala, M., Hayden, B., "Design of the master optical reference for the James Webb Space Telescope," Proc. SPIE 9143, 914306 (2014).

[16] Yang, K., Glazer, S. D., Thomson, S. R., Feinberg, L. D., Burt, W., Comber, B. J., Ousley, W., Franck, R., "Thermal Model Performance for the James Webb Space Telescope OTIS Cryo-Vacuum Test," $48^{\text {th }}$ International Conference on Environmental Systems, ICES-2018-35 (2018).

[17] Atkinson, C., Arenberg, J., Matthews, G., Waldman, M., Wertheimer, A., Whitman, T., Oschmann, J., “Architecting a revised optical test approach for JWST," Proc. SPIE 7010, 70100Q (2008).

[18]Feinberg, L. D., Barto, A., Waldman, M., Whitman, T., "James Webb Space Telescope system cryogenic test plans," Proc. SPIE 8150, 815007 (2011).

[19] Feinberg, L. D., Keski-Kuha, R., Atkinson, C., Texter, S. C., "Use of a pathfinder optical telescope element for James Webb Space Telescope risk mitigation,” Proc. SPIE 7731, 77313T (2010).

[20] Feinberg, L. D., Keski-Kuha, R., Atkinson, C., Booth, A., Whitman, T., "James Webb Space Telescope (JWST) Optical Telescope Element (OTE) Pathfinder status and plans,” Proc. SPIE 9143, 91430E (2014).

[21] Lunt, S. R., Rhodes, D., DiAntonio, A., Boland, J., Wells, C., Gigliotti, T., Johanning, G., "Model predictions and observed performance of JWST’s cryogenic position metrology system," Proc. SPIE 9904, 99044C (2016). 
[22] Hadaway, J. B., et al., "Performance of the primary mirror center-of-curvature optical metrology system during cryogenic testing of the JWST Pathfinder telescope," Proc. SPIE 9904, 99044E (2016).

[23] Whitman, T. L., Wells, C., Hadaway, J. B., Knight, J. S., Lunt, S., "Alignment test results of the JWST Pathfinder Telescope mirrors in the cryogenic environment," Proc. SPIE 9904, 990449 (2016).

[24] Wells, C., et al., "Characterization of the JWST Pathfinder mirror dynamics using the center of curvature optical assembly (CoCOA)," Proc. SPIE 9904, 990440 (2016).

[25] Smith, K. Z., Acton, D. S., Gallagher, B. B., Knight, J. S., Dean, B. H., Jurling, A. S., Zielinski, T. P., "Calibration results using highly aberrated images for aligning the JWST instruments to the telescope," Proc. SPIE 9904, 990442 (2016).

[26] Warden, R. M., "Cryogenic Nano-actuator for JWST", Proceedings of the 38th Aerospace Mechanisms Symposium, Langley Research Center (2006).

[27] Howard, J. M., Ha, K. Q., West, G. J., Smith, J. S., Carnahan, T. M., "JWST structural-thermal-optical stability model validation," Proc. SPIE 10705, in press (2018).

[28] Atkinson, C., Feinberg, L., Carnahan, T., Knight, S., "Thermal distortion measurements of the JWST Optical Telescope Element," Proc. SPIE 10698, in press (2018).

[29] Wolf, E. M., et al., "JWST mirror and actuator performance at cryo-vacuum," Proc. SPIE 10698, in press (2018).

[30] Vila Costas, M. B., et al., "JWST cryo fine guidance closed loop test results," Proc. SPIE 10698, in press (2018).

[31] Glazer, S. D., Yang, K., Comber, B. J., Ousley, W., Cleveland, P. E., "Off-Nominal Planning for the Cryogenic Thermal Vacuum/Thermal Balance Test of the James Webb Space Telescope Optical Telescope Element/Integrated Science Instrument Module at the Johnson Space Center," $48^{\text {th }}$ International Conference on Environmental Systems, ICES-2018-283 (2018).

[32] te Plate, M., et al., "JWST's near-infrared spectrograph status and first OTIS test results," Proc. SPIE 10698, in press (2018).

[33] Lightsey, P. A., et al., "James Webb Space Telescope optical performance predictions post cryogenic vacuum tests," Proc. SPIE 10698, in press (2018).

[34] Cosentino, J., et al., "Setting the James Webb Space Telescope primary mirror radius of curvature and conic constant during cryogenic testing," Proc. SPIE 10698, in press (2018).

[35] Knight, J. S., Feinberg, L., Howard, J., Acton, D. S., Whitman, T. L., Smith, K., "Hartmann test for the James Webb Space Telescope," Proc. SPIE 9904, 99040C (2016).

[36] Coyle, L. E., Chonis, T. S., Smith, K. Z., Knight, J. S., "Optical assessment of the James Webb Space Telescope primary mirror and secondary mirror cryogenic alignment with a Hartmann test," Proc. SPIE 10706, in press (2018).

[37] Acton, D. S., Knight, J. S., Chonis, T. S., Coyle, L. E., Smith, K., Coppock, E., "Wavefront sensing and controls demo during the cryo-vac testing of JWST," Proc. SPIE 10698, in press (2018).

[38] Lajoie, C.-P., et al., "Wavefront sensing and controls demo during the cryo-vacuum test of JWST: exercising the science and operations center," Proc. SPIE 10698, in press (2018). 